\title{
Application of SN-EMD in Mode Feature Extraction of Ship Radiated Noise
}

\author{
Fang Niu $\mathbb{D}^{1,2,3}$ Juan Hui $\mathbb{D}^{1,{ }^{1,2,3}}$ Anbang Zhao $\mathbb{D}^{1,2,3,4}$ Yue Cheng, ${ }^{1,2,3}$ and Yang Chen \\ ${ }^{1}$ Acoustic Science and Technology Laboratory, Harbin Engineering University, Harbin 150001, China \\ ${ }^{2}$ Key Laboratory of Marine Information Acquisition and Security, Harbin Engineering University, Ministry of Industry and Information \\ Technology, Harbin 150001, China \\ ${ }^{3}$ College of Underwater Acoustic Engineering, Harbin Engineering University, Harbin 150001, China \\ ${ }^{4}$ National Key Laboratory of Science and Technology on Underwater Acoustic Antagonizing, China State Shipbuilding Corporation \\ Systems Engineering Research Institute, Beijing 100036, China \\ ${ }^{5}$ Department of Information Science and Engineering, Changzhou University, Changzhou 213164, China
}

Correspondence should be addressed to Juan Hui; huijuan@hrbeu.edu.cn

Received 24 August 2018; Revised 4 November 2018; Accepted 22 November 2018; Published 3 December 2018

Academic Editor: Juan P. Amezquita-Sanchez

Copyright @ 2018 Fang Niu et al. This is an open access article distributed under the Creative Commons Attribution License, which permits unrestricted use, distribution, and reproduction in any medium, provided the original work is properly cited.

\begin{abstract}
Due to the randomness of added noise, noise-assisted versions based on EMD (empirical mode decomposition) usually cause new "mode mixing" problem. In addition, these algorithms also have problems such as high time-consuming and large recovering error. For the reasons, a new method SN-EMD (Selective Noise-assisted EMD) is put forward in this paper. It determines whether to add noise as assistance by judging whether there is high frequency intermittent component contained in the signal or not. The new method was proved to have the optimal performance by comparing the performance parameters for evaluating the decomposition. In this paper, SN-EMD was used to decompose ship radiated noise. On account of the differences in the original information contained in each mode of radiated noise signals from different ship, we selected the first three modes for processing. Average instantaneous frequency, center frequency, energy density, and energy distribution ratio were extracted as mode feature of ship targets for classification and recognition. Spatial distribution of the feature quantities in three-dimensional space verified similarity of the same target and separability of different targets.
\end{abstract}

\section{Introduction}

EMD [1] is an adaptive signal processing method proposed by N. E. Huang, which is especially suitable for nonlinear and nonstationary signal. It can decompose the original signal in an adaptive manner in fast and slow oscillations based on the direct extraction of signals with different time scale. The original signal can be decomposed into a series of monocomponent signals and a component in a monotonic trend. These monocomponent signals are usually referred to as "intrinsic modal functions (IMFs)" (or modes). Although the modes obtained by EMD are local narrowband and can represent the actual physical process, therefore, analysis of instantaneous frequency by Hilbert transform can be performed for each mode.
The real signal is complex, and intermittency of frequency algorithm causes mode mixing in EMD. Namely, signal components on different time scales are decomposed into a mode, or signal components at the same time scale are decomposed into different modes. In order to solve the problem of mode mixing, based on the EMD algorithm, a series of noise-assisted algorithms named Ensemble Empirical Mode Decomposition (EEMD) [2], Complementary Ensemble Empirical Mode Decomposition (CEEMD) [3], Complete Ensemble Empirical Mode Decomposition with Adaptive Noise (CEEMDAN) [4], and improved CEEMDAN [5] were proposed consecutively. Among them, the improved CEEMDAN has the best results. There is no problem that the number of modes is different due to the additive noise in EEMD and CEEMD, and the problem of false modes in 
CEEMDAN. However, the improved CEEMDAN still has aspects for improvement: (I) time-consuming issues: in order to reduce the residual error, it needs hundreds of decomposition over an ensemble of noisy copies of the original signal, so the algorithm is time-consuming; (II) the problem of mode mixing: for the reason of added noise, this algorithm can avoid decomposing the components on different time scales into the same mode, but there is still a second type of mode mixing phenomenon that the components at the same time scale are decomposed into different modes. To solve the two problems above, we introduce an improved algorithm SNEMD.

The decomposition algorithms have applications in many fields, such as electrocardiograph (ECG) signal denoising and remote monitoring of the respiration and heartbeat of a patient $[5,6]$, time-frequency analysis of seismic signals $[7,8]$, analysis of the regional features of temperature variation trends $[9,10]$, electrical filed such as power quality estimation $[11,12]$, mechanical fault diagnosis of roller bearings $[13,14]$, polymer electrolyte membrane fuel cell fault diagnosis in chemistry [15], visual enhancement of underwater images, and nonlinear feature extraction in underwater acoustics $[16,17]$. Though the actually ship radiated noise is Gaussianlike in terms of probability distribution [18], it contains many definitive ingredients. The center frequency distribution of the modes of Gaussian noise usually obeys the simple bisection rule, while ship radiated noise does not $[19,20]$. The difference of center frequency distribution of modes reflects the characteristics of different types of ship. In addition, the energy distribution of the mode of each order also has its own rules. As a result, the features such as instantaneous frequency and instantaneous amplitude extracted from each mode can be used as the basis for classification and identification of ship targets.

This paper is organized as follows: Section 2 introduces the existing empirical mode decomposition algorithms and the new algorithm SN-EMD. Section 3 compares the performance of these decomposition algorithms. In Section 4, instantaneous frequency and other factures are extracted from modes of real ship radiation noise data, and their separability is verified in a three-dimensional space. The conclusions are drawn in Section 5.

\section{Methods}

\subsection{Existing Methods}

2.1.1. EMD. The EMD algorithm can be described as follows.

Step 1. Set $k=0$ and $r_{k}(t)=x(t)$.

Step 2. Find all the maxima (minima) of $r_{k}(t)$. Interpolate between maxima (minima) of $r_{k}(t)$ to obtain the upper (lower) envelope $e_{\max }(t)\left(e_{\min }(t)\right)$.

Step 3. Compute the mean envelope $m(t)=\left(e_{\max }(t)+\right.$ $\left.e_{\min }(t)\right) / 2$.

Step 4. Compute the IMF candidate $c_{k+1}(t)=r_{k}(t)-m(t)$.
Step 5. Judge whether $c_{k+1}(t)$ is an IMF.

(i) Yes. Set $i m f_{k+1}(t)=c_{k+1}(t)$ and save $i m f_{k+1}(t)$ as IMF of order $k+1$. Compute the residue $r_{k+1}(t)=x(t)-$ $\sum_{n=1}^{k+1} i m f_{k+1}(t)$, do $k=k+1$, and treat $r_{k}(t)$ as input data in Step 2.

(ii) No. Treat $c_{k+1}(t)$ as input data in Step 2.

Step 6. Continue until the final residue $r_{k}(t)$ satisfies some predefined stopping criterion.

The original signal $x(t)$ can be expressed as a sum of $\operatorname{imf}_{n}(t),(n=1, \ldots, N)$.

There is mode mixing problem in EMD decomposition, which makes it impossible for EMD to achieve separation of components of the signal at different frequency. The mode obtained by decomposition cannot reflect the instantaneous frequency characteristics of the signal and fundamentally loses the original intention of EMD algorithm. Meanwhile, the deficiency limits the engineering application of the algorithm.

2.1.2. EEMD. In order to solve the problem of mode mixing, $\mathrm{Wu}, \mathrm{Z}$. [2] proposed the EEMD algorithm. EEMD defines the "true" modes as the average of the corresponding IMFs obtained from an ensemble of the original signal plus different realizations of finite variance white noise. On the basis of the characteristics of the EMD's binary filter bank, it reduces mode mixing by filling the entire time-frequency space, with adding white noise to the signal.

EEMD algorithm also has some drawbacks: (I) Different realizations of finite variance white noise may produce different numbers of modes; (II) the average mode may not meet the requirements of effective IMF; (III) when IMFs are used to reconstruct the signal, the residual noise cannot be ignored; (IV) ensemble average in EEMD usually needs operation for several hundred times, which is very timeconsuming.

2.1.3. CEEMD. Yeh, J.R. [3] proposed the CEEMD algorithm, which is different with EEMD in that the noise added to the original data is in pairs (a positive number and a negative number). Adding noise with a pair of opposite signs makes the residual noise basically cancel out each other and achieve the effect of "complementary."

2.1.4. CEEMDAN. CEEMDAN proposed in [4] is an important improvement to EEMD. The first-order mode using CEEMDAN algorithm is the same as that using EEMD, and other signal modes are the first-order mode decomposed from the residual signal plus white noise mode in the same order. Also, the coefficients of each noise mode are all adaptive, which are related to the residual signal and noise mode. The improvement of CEEMDAN reduces the reconstruction error and solves the problem that different realizations of signal plus noise might produce different number of modes. 
2.1.5. Improved CEEMDAN. In order to reduce the residual noise, Colominas, M.A. [5] made an improvement on CEEMDAN: estimating local mean instead mode. From the firstorder mode, the noise added in the signal is mode component decomposed from white noise rather than the original white noise.

2.2. $S N-E M D$. When there are high frequency intermittent components contained in the signal, the EMD will lead to the first type of mode mixing problem that signal components on different time scales in one mode. In order to solve the problem, a series of noise-assisted versions based on EMD have been proposed. However, due to the randomness of the added noise, the noise-assisted algorithms may cause the second type of mode mixing phenomenon that the signal components on the same time scale are decomposed into different modes. In addition, these algorithms also have problems such as high time-consuming and large residual error. For the reasons, a new method SN-EMD (Selective Noiseassisted EMD) is proposed in this paper. Therefore, in order to avoid the phenomenon of mode mixing, it is necessary to judge whether high frequency intermittent component is contained before decomposition and then decide whether to add noise for decomposition or not.

At the high frequency intermittent component of the signal, the interval time between the extrema of the signal envelope will decrease obviously. That is, at the beginning and the end of the intermittent component, the interval time between the extrema has a jump phenomenon. By detecting fluctuation of interval time between the extrema, whether there is high frequency intermittent component in the signal can be judged. In this paper, variance of interval time is used to judge whether there is intermittent component.

Let $M(\cdot)$ be the operator which produces the local mean of the signal that is applied to. $\langle\cdot\rangle$ indicates the action of averaging operation for $I$ times instead of $(1 / I) \sum_{i=1}^{I / 2}(\cdot)$. $\omega^{(i \pm)}(t)$ is the realizations of additive white noise mode in pairs for the $i$ th time.

We propose the SN-EMD algorithm as follows.

Step 1. Set $k=0$ and $r_{k}(t)=x(t)$.

Step 2. Judge whether high frequency intermittent component is contained in $r_{k}(t)$.

(i) Yes. Calculate the local means of $r_{k}{ }^{(i \pm)}(t)=r_{k}(t)+$ $\beta_{k}\left[\omega_{k+1}{ }^{(i \pm)}(t)\right]$ by EMD to obtain the residue $r_{k+1}(t)=$ $\left\langle M\left(r_{k}{ }^{(i \pm)}(t)\right)\right\rangle$ and go to Step 3.

(ii) No. Get the $k+1$ th mode $i m f_{k+1}(t)$ and the residue $r_{k+1}(t)=r_{k}(t)-i m f_{k+1}(t)$ by EMD and go to Step 4 .

Step 3. Get the $k+1$ th mode $\widetilde{i m f_{k+1}}(t)=r_{k}(t)-r_{k+1}(t)$.

Step 4. Go to Step 2 and continue for next $k$ until residue satisfies predefined stopping criterion.

Step 5. Save all the modes from Steps 2 and 3. The original signal $x(t)$ can be expressed as a sum of $\operatorname{imf} f_{n}(t), \quad(n=$ $1, \ldots, N)$ and $r_{N}(t)$.
Coefficient $\beta_{k}\left(\beta_{k}=\varepsilon_{k} s t d\left(r_{k}(t)\right) / s t d\left(\omega_{k+1}^{(i \pm)}(t)\right)\right)$ controls the amplitude of added noise. $\beta_{k}$ is chosen to obtain a desired SNR between the added noise and the residue to which the noise is added. $\varepsilon_{k}$ is a constant not greater than 0.2 . Figure 1 gives the flowchart of SN-EMD.

\section{Simulation and Performance Analysis}

In this section, two artificial signals are decomposed by several decomposition algorithms in Section 2. The performance of these algorithms is compared and analyzed based on the evaluating indicator given.

3.1. Quantitative Evaluation Indexes. In order to accurately describe the performance of the mode decomposition algorithm, five quantitative evaluation indexes for mode separation are introduced: Consuming Time $(C T)$, Index of Orthogonality (IO), Similarity Index (SI), and Residual Error (RE).

IO describes the orthogonality between different modes, which can examine whether there is mode mixing between every two modes. The ideal IMF components are completely orthogonal, while $I O$ is equal to zero. Conversely, the larger the $I O$, the more severe the mode mixing of the two IMF components. In order to ensure $I O$ positive, the definition given in [19] is modified to

$$
I O=\sum_{j=1}^{N} \sum_{i=1}^{N}\left|\sum_{l=1}^{L} \frac{i m f_{i}(l) i m f_{j}(l)}{x^{2}(l)}\right|
$$

$i m f_{i}(l)$ and $i m f_{j}(l)$ are, respectively, the discrete sequences of the $i$ th and the $j$ th mode $(i \neq j)$, and $x(l)$ is in the discrete form of $x(t)$. The following signals and modes are mostly represented in discrete form. The length of the signal sequence is $L$ and the highest order of the IMF is $N$.

SI describes the similarity between the each order IMF and the original signal. It is used to measure whether the mode decomposition algorithm truly separates the modes from the original signal. SI is defined as follows:

$$
S I=\frac{1}{N} \sum_{n=1}^{N} c\left(x(l), i m f_{n}(l)\right)
$$

The larger the SI, the more similar the IMF and the original signal. $\widetilde{x(l)}$ indicates the mean value of the original input signal sequence. The correlation coefficient expression is as follows:

$$
\begin{aligned}
c\left(x(l), i m f_{n}(l)\right) & =\frac{\sum_{l=1}^{L}[x(l)-\widetilde{x(l)}]\left[i m f_{n}(l)-i \widetilde{m f_{n}(l)}\right]}{\sqrt{\sum_{l=1}^{L}[x(l)-\widetilde{x(l)}]^{2}} \sqrt{\sum_{l=1}^{L}\left[i m f_{n}(l)-\widetilde{i m f_{n}(l)}\right]^{2}}}
\end{aligned}
$$

$R E$ represents residual error of noise when recovering signal components. Several noise-assisted decomposition algorithms require that the residual noise be reduced by 


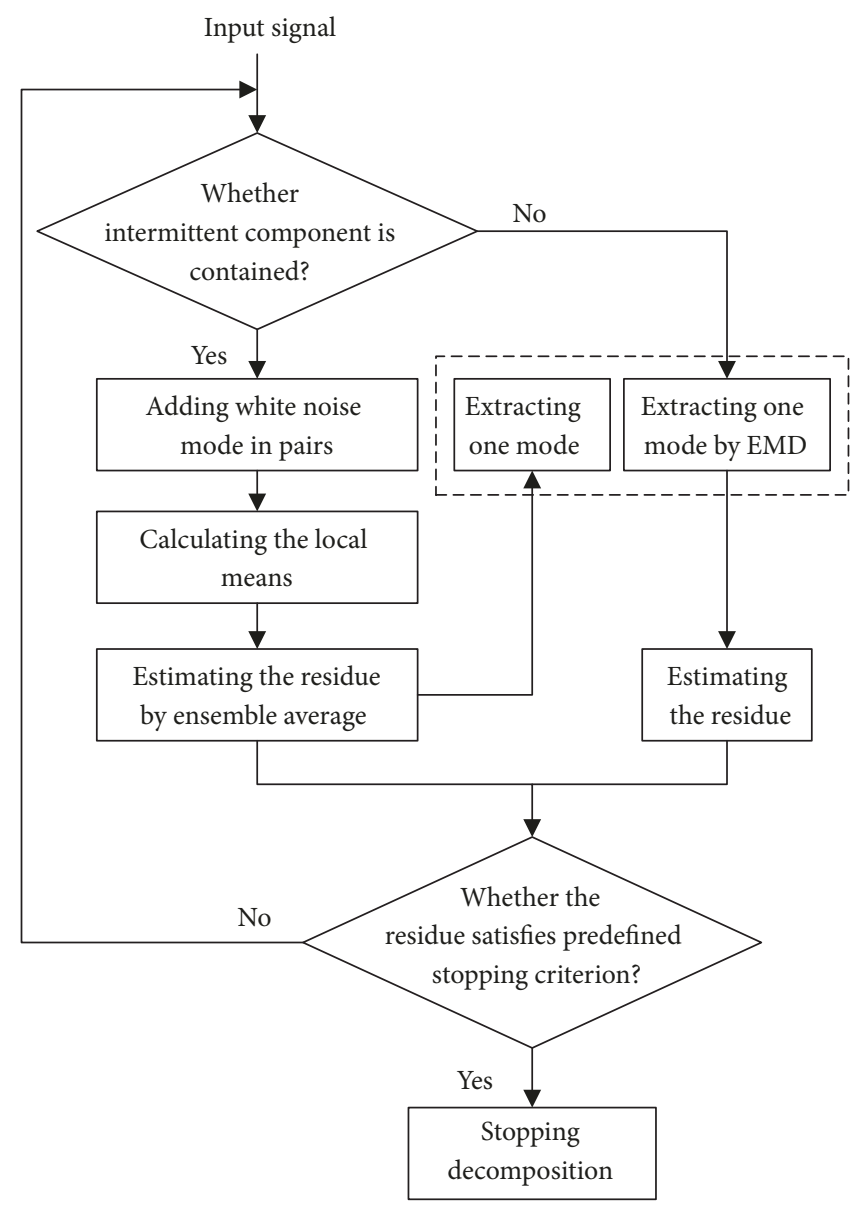

FIGURE 1: SN-EMD algorithm flowchart.

averaging for many times. The residual error when recovering $x_{1}(t)$ from artificial signal $x(t)$ above can be expressed as

$$
R E_{1}=\frac{\sum_{l=1}^{L}\left[i m f_{1}(l)-x_{1}(l)\right]^{2}}{\sum_{l=1}^{L}\left[i m f_{1}(l)\right]^{2}}
$$

3.2. Simulation. As an example, we propose here a classical mode mixing example. The input signal is an intermittent high frequency sine component plus a sustained intermediate frequency component and a low frequency component for 1 second. The artificial input signal $x(t)=x_{1}(t)+x_{2}(t)+x_{3}(t)$ is shown in Figure 2. Intermittent high frequency signal is expressed as $x_{1}(t)=0.2 \sin (2 \pi 300 t)(0.3 \leq t \leq 0.35$, $0.6 \leq t \leq 0.65,0.8 \leq t \leq 0.85)$; two sustained sine signals are $x_{2}(t)=\sin (2 \pi 65 t)(0 \leq t \leq 1)$ and $x_{3}(t)=$ $0.3 \sin (2 \pi 15 t)(0 \leq t \leq 1)$.

In order to determine whether there is high frequency intermittent component in the input signal, the maximum and minimum values of the signal are marked first, and the time interval between the extrema is calculated. The maximum and minimum intervals are normalized to calculate the respective variances. The average value of the two variances is used as the final judgment parameter. Considering the divergent effect of the boundary in decomposition, the first two extrema and the last two extrema are discarded, so the number of extreme points is usually no less than 6 . In this paper, the threshold is calculated from decomposition of white noise. Mean variances of normalized interval time between the extrema of white noise and each residue are calculated. The mean value of the several mean variances multiplied by 1.5 is set to be the final threshold, which is equal to 0.032 . When the judgment parameter is greater than 0.032 , the signal is considered to contain intermittent component.

Figure 3 shows the time interval between the extrema of the signal when there is and there is no intermittent component $x_{1}(t)$, respectively. The judgment parameters are, respectively, 0.11 and 0.0019 when there is and there is no intermittent component. The threshold is proven to be effective.

Decompose the artificial signal separately using the six algorithms in Section 2 and the IMFs are shown in Figures 4(a)-4(f), with ensemble sizes of $I=100$ and coefficient $\varepsilon_{n}=0.05$.

From the comparison of Figures 4(a)-4(f), it can be found that, in the first-order mode decomposed by EMD, the intermediate frequency component $x_{2}(t)$ is filled in the gap of high frequency component $x_{1}(t)$. In other words, using the EMD algorithm, the high frequency intermittent component cannot be extracted from the original signal. 

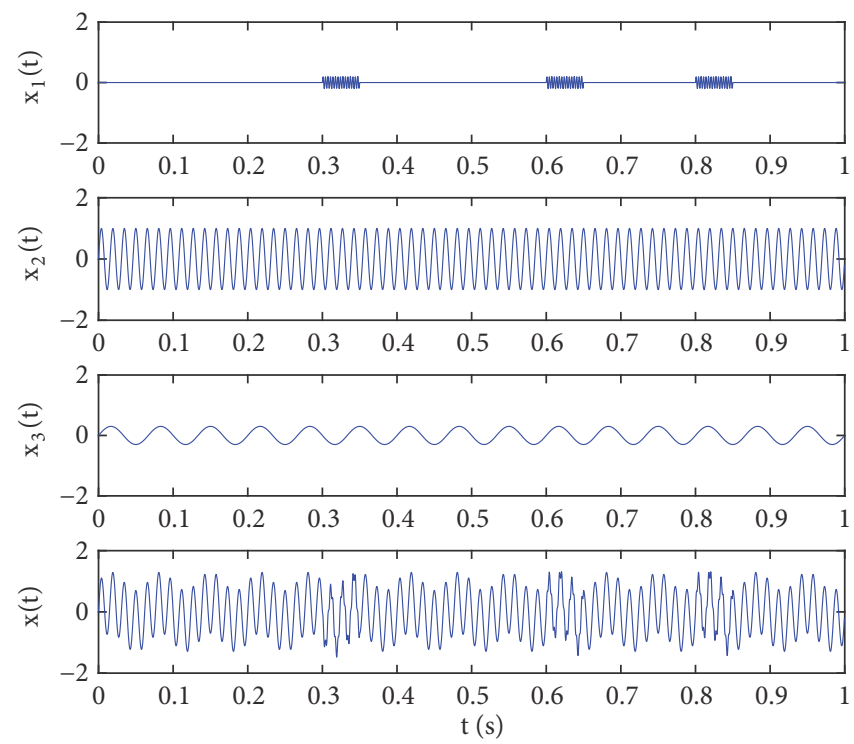

FIgURe 2: Artificial signal.

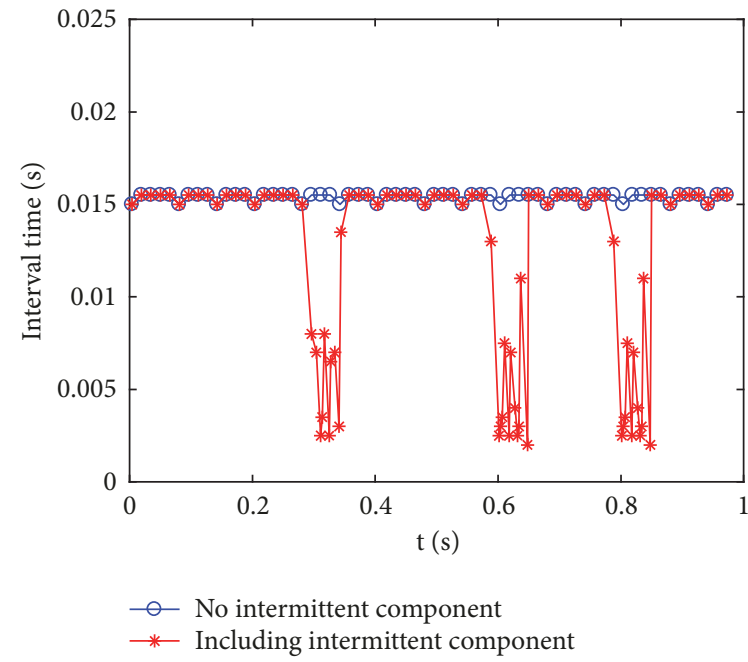

(a)

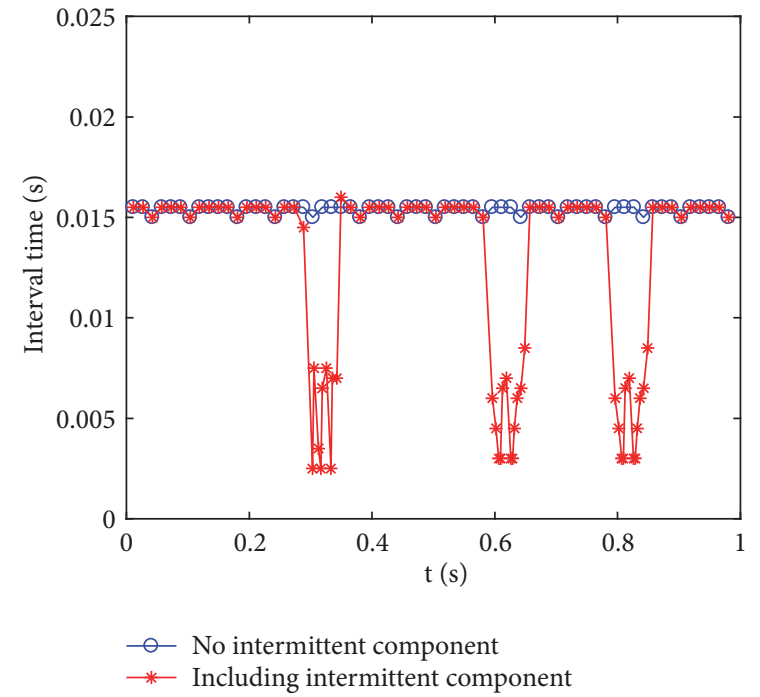

(b)

Figure 3: Interval time between (a) maxima and (b) minima.

Frequency mixing appears when extracting low-frequency component $x_{3}(t)$ by EEMD and CEEMD; that is, a time scale signal component is decomposed into different modes. Not only mode mixing phenomenon but also false modes such as the second and fifth IMFs appear in decomposition by CEEMDAN as shown in Figure 4(d). Further, the improved CEEMDAN still has mode mixing problems. However, the SN-EMD algorithm proposed in this paper can accurately decompose the original signal into three components, solving the problem of mode mixing. The evaluation indexes $C T, I O$, and SI are counted and listed in Table 1. Of these, for the five noise-assisted algorithms, the ensemble average number of times $I$ is 100 .
Comparing the evaluation indexes in Table 1, it can be seen that the SN-EMD algorithm has the smallest $I O$ and the largest $S I$, which shows the new algorithm has a better performance of extracting true modes from the signal. In addition, CT of SN-EMD is much smaller than other noiseassisted version.

When using the noise-assisted method, due to added random noise, there is residual noise in each mode, which generally decreases with the increase of the average number of times. The ensemble average number of times $I$ is taken as 20,100,200, and 400, respectively, and we can get the average residual error of 100 calculations. For the reason of mode mixing, the true mode can be seen as the 
TABLE 1: Performance statistics of mode composition algorithms.

\begin{tabular}{lcccccc}
\hline & EMD & EEMD & CEEMD & CEEMDAN & Improved CEEMDAN & SN-EMD \\
\hline$C T / \mathrm{s}$ & 0.23 & 13.49 & 12.97 & 36.04 & 29.42 & 0.31 \\
$I O$ & 0.19 & 0.11 & 0.10 & 0.41 & 0.056 \\
$S I$ & 0.26 & 0.32 & 0.32 & 0.28 & 0.34 & 0.37 \\
\hline
\end{tabular}

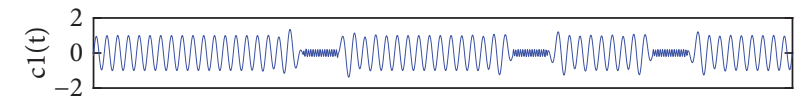

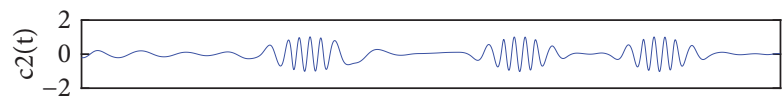

苟

䒿 0.5

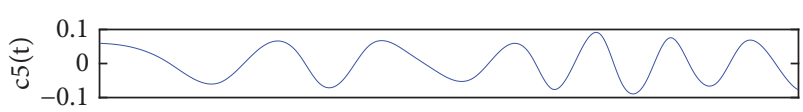

$\overbrace{0.0}^{0.1} \begin{array}{r}0.1 \\ 0\end{array}$

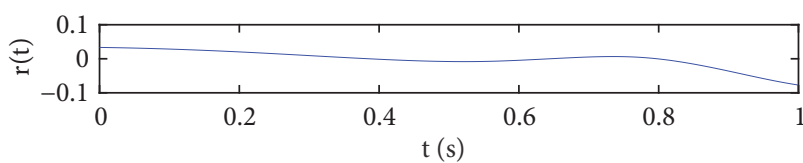

(a)

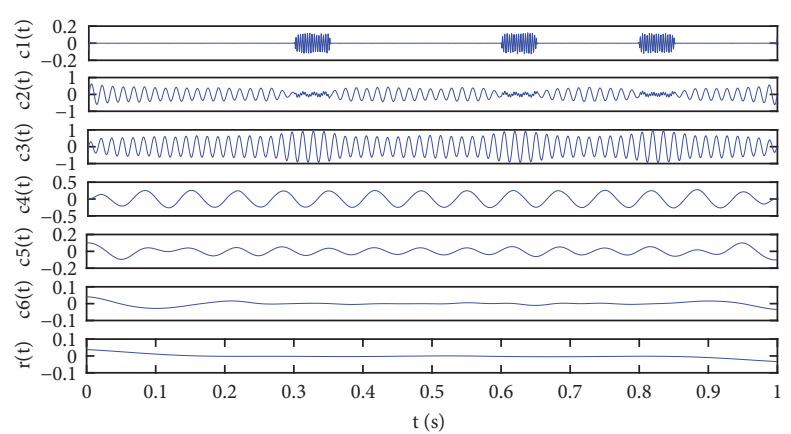

(c)

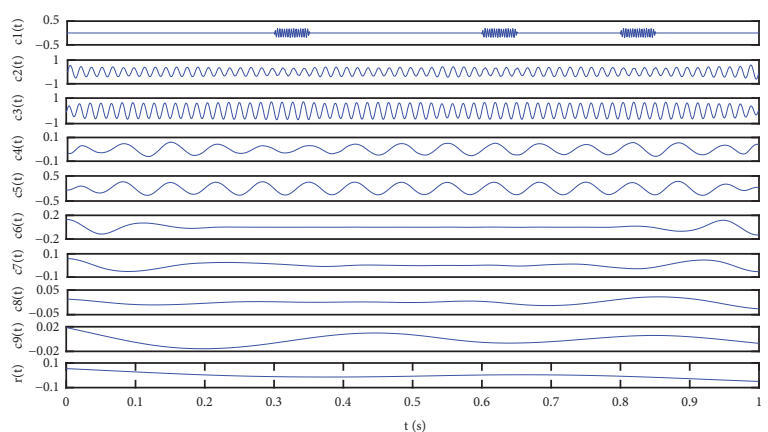

(e)

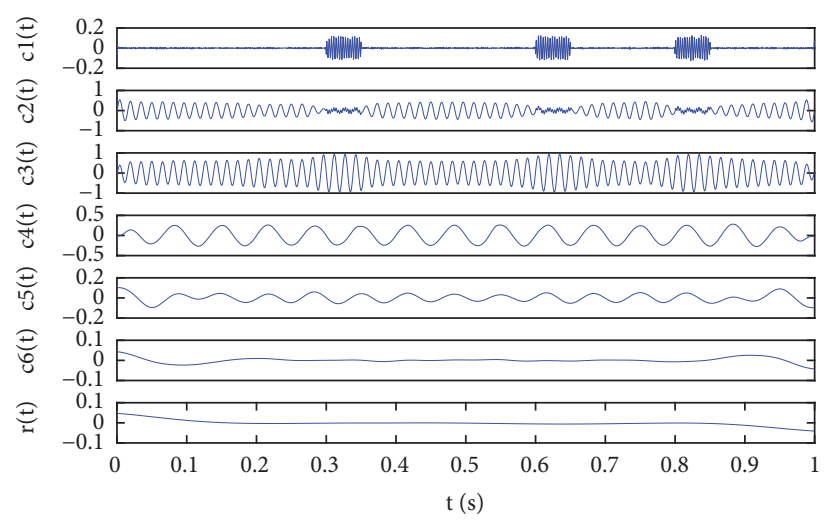

(b)

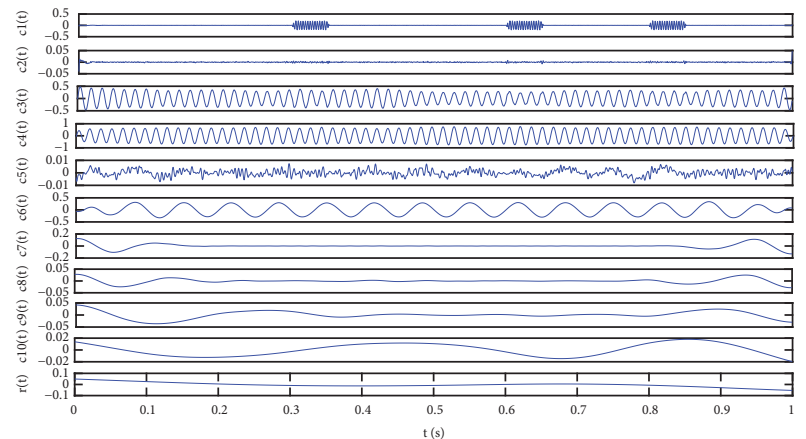

(d)

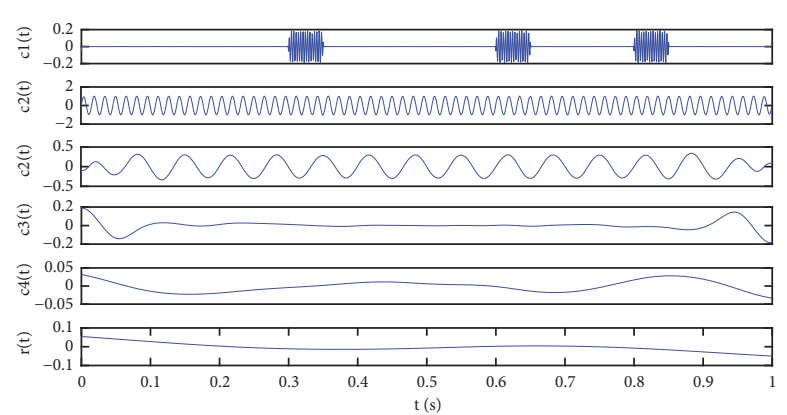

(f)

FIGURE 4: Decomposition of artificial signal by (a) EMD, (b) EEMD, (c) CEEMD, (d) CEEMDAN, (e) improved CEEMDAN, and (f) SNEMD. 


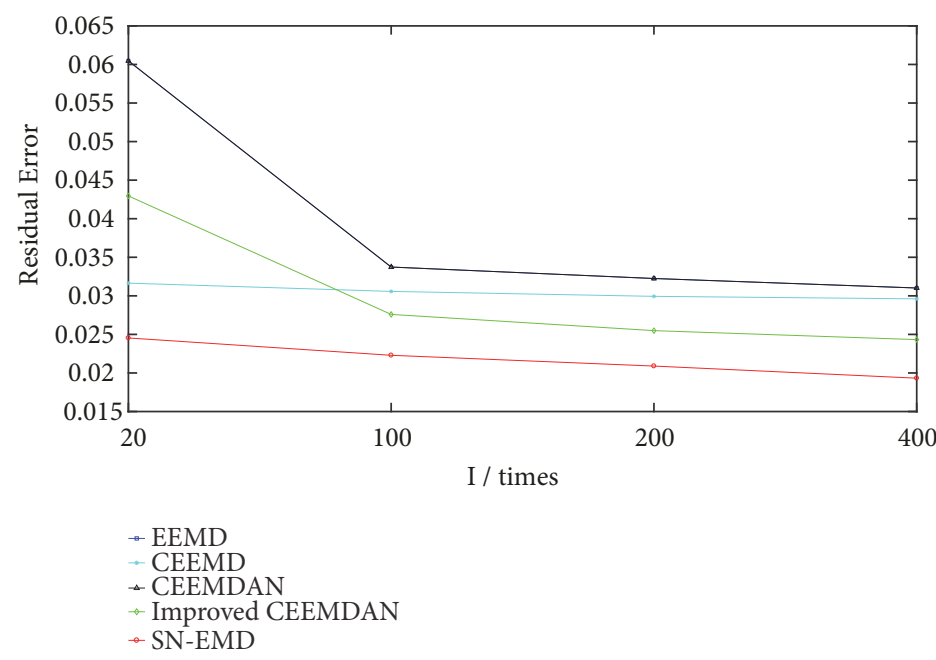

(a)

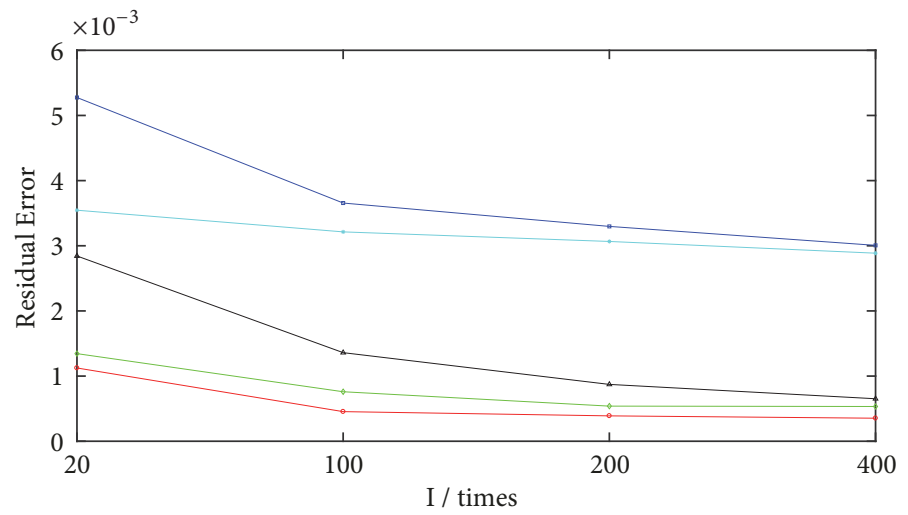

- EEMD

CEEMD

- CEEMDAN

- Improved CEEMDAN

- SN-EMD

(b)
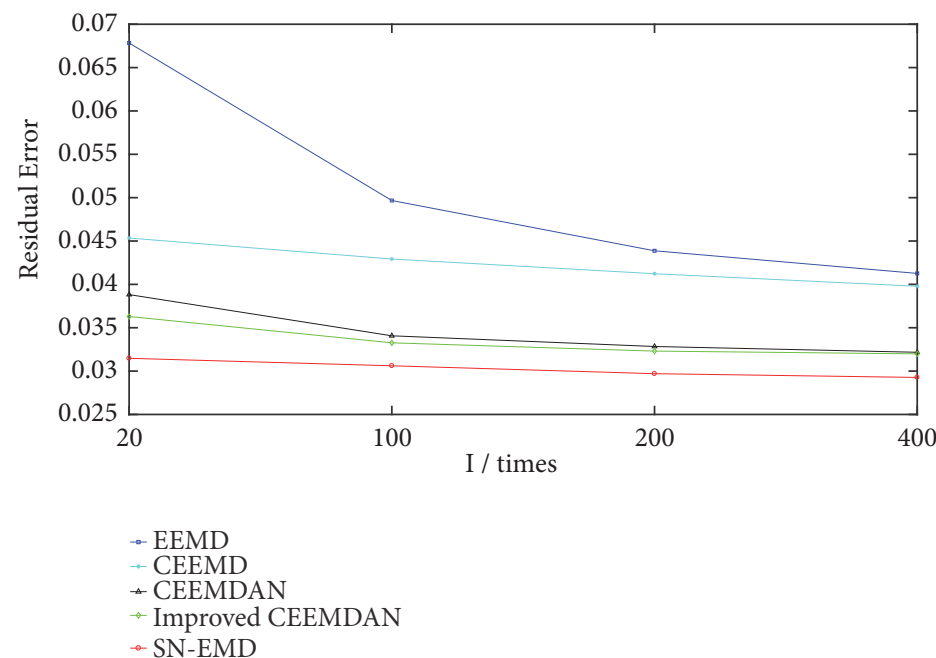

(c)

FIGURE 5: Residual error when recovering (a) $x_{1}(t)$, (b) $x_{2}(t)$, and (c) $x_{3}(t)$. 
TABLE 2: Performance statistics of mode composition algorithms.

\begin{tabular}{lcccccc}
\hline & EMD & EEMD & CEEMD & CEEMDAN & Improved CEEMDAN & SN-EMD \\
\hline$C T / \mathrm{s}$ & 0.29 & 15.47 & 15.14 & 38.40 & 33.67 & 0.44 \\
IO & 0.38 & 0.31 & 0.27 & 0.64 & 0.88 & 0.32 \\
SI & 0.24 & 0.28 & 0.29 & 0.22 & 0.35 \\
\hline
\end{tabular}

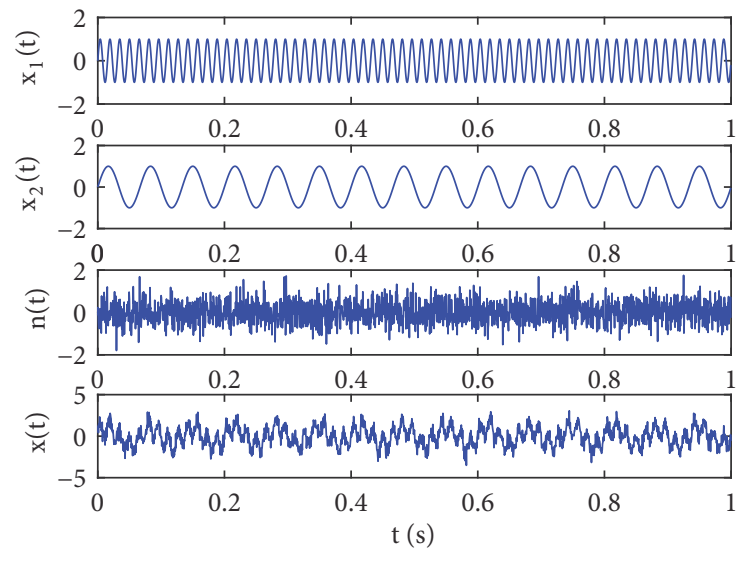

Figure 6: Artificial signal with noise.

superimposition of mode components in the same time scale, which can be judged based on the orthogonality between two modes. The 3rd to 5th order modes, as decomposed by the EEMD algorithm in Figure 4(b), are in a time scale, which can be superimposed as the mode corresponding to $x_{3}(t) . R E_{i}$ represents the residual error for recovering $x_{i}(t)$. Figures 5(a)-5(c) show the comparison of the residual error in recovering the three components of the original signal.

As can be seen from Figures 5(a)-5(c), the residual noise error by SN-EMD when recovering the three components is less than that by the other four algorithms. Furthermore, only if the ensemble average number of times $I$ is taken as 20, the residual noise error has been quite slow. So SNEMD can reduce the average number of times and save the operation time. In a word, the new algorithm SNEMD integrates the advantages of the existing decomposition methods.

To research the decomposition with noise, another example is proposed. The input signal is composed of two sine components and noise. The artificial input signal $x(t)=$ $x_{1}(t)+x_{2}(t)+n(t)(0 \leq t \leq 1)$ is shown in Figure 6 . The high frequency sine signal is expressed as $x_{1}(t)=\sin (2 \pi 65 t)$, another sine signal is $x_{2}(t)=\sin (2 \pi 15 t)$, and $n(t)$ is filtered Gaussian noise by a $500 \mathrm{~Hz}$ low-pass filter.

Decompose the artificial signal separately using the six algorithms and the modes are shown in Figures $7(\mathrm{a})-7(\mathrm{f})$, with ensemble sizes of $I=100$ and coefficient $\varepsilon_{n}=0.05$.
Obviously, the noise $n(t)$ leads to mode mixing problems marked in Figure 7(a) with red boxes. Furthermore, high frequency component and low frequency component alternately appear in. $c_{2}(t)$. From Figures $7(\mathrm{~b})-7(\mathrm{e})$, it can be found that mode mixing, false mode extracting, and other problems cannot be avoided when using the four existing noise-assisted decomposition algorithms. However, the SN-EMD algorithm solves these problems as shown in Figure 7(f). The mean variances of normalized interval time between the maxima and minima of $x(t)$ and $r_{i}(t)(i=1, \ldots, 4$, for the number of maxima of $r_{5}(t)$ being less than 6$)$ are $0.017,0.076,0.0071$, 0.0017 , and 0.024 , respectively. Only 0.076 is greater than the threshold 0.032 , so high frequency intermittent component firstly appears in $r_{1}(t)$. The interval time between the extrema of $r_{1}(t)$ is shown in Figure 8.

Comparing the evaluation indexes shown in Table 2, it can be seen that the SN-EMD algorithm has a better performance of extracting true modes from the signal. In addition, the noise degrades the decomposition performance of each algorithm.

The ensemble average number of times $I$ is taken as $20,100,200$, and 400, respectively, and we can get the average residual error of 100 calculations. Figure 9 shows the comparison of the residual error when recovering the two signal components $x_{1}(t)$ and $x_{2}(t)$. In Figure 8 , it can be seen that the residual noise error by SN-EMD when recovering the three components is less than that by the other four algorithms.

\section{Experimental Results and Discussion}

The mode decomposition of the ship radiated noise is to adaptively divide the original signal according to different time scales. The original information contained in the signal is also assigned to each mode when decomposing the signal. For different types of ships, the distribution of the original information contained in each mode is different. Based on these differences, time-frequency features can be extracted for each mode as a standard for classification and recognition. The flowchart of mode feature extraction from ship radiated noise based on SN-EMD is shown in Figure 10.

\subsection{Mode Feature}

4.1.1. Average Instantaneous Frequency. The average instantaneous frequency can exhibit the frequency distribution of a mode. The instantaneous frequency $f_{i j}$ at the moment of $j$ can be achieved by taking the derivative of the instantaneous phase of the $i$ th mode obtained with Hilbert transform. Set 


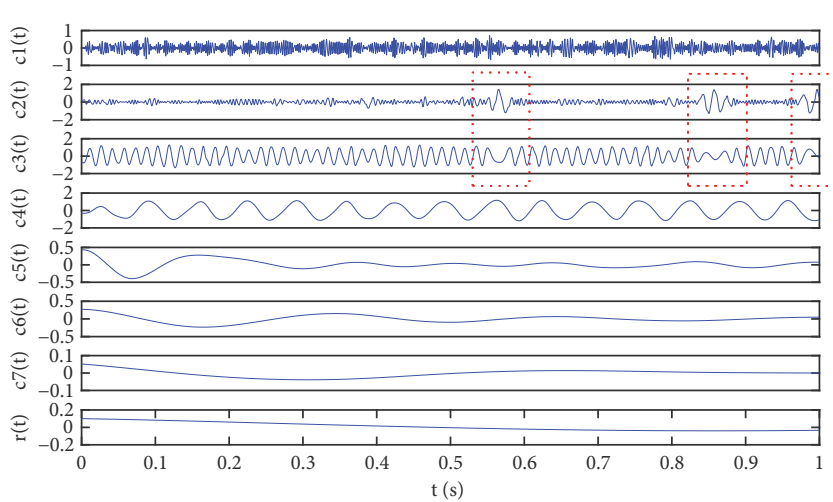

(a)

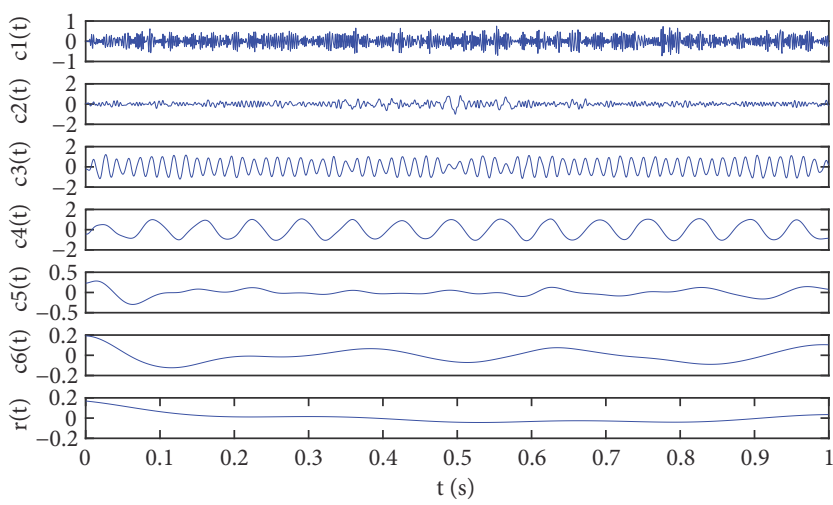

(c)

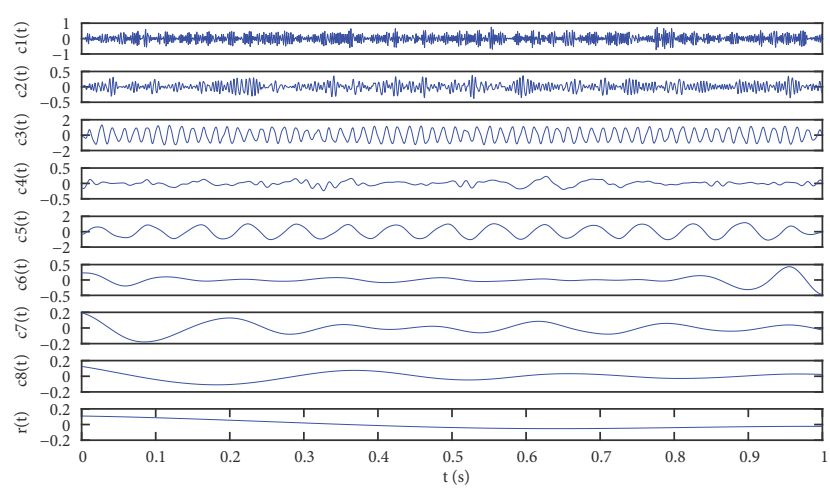

(e)

FIguRE 7: Decomposition of artificial signal with noise by (a) EMD, (b) EEMD, (c) CEEMD, (d) CEEMDAN, (e) improved CEEMDAN, and (f) SN-EMD.

$K$ to be the number of sample points per unit time. So, the average instantaneous frequency per unit time is

$$
\overline{f_{i}}=\frac{1}{K} \sum_{j=1}^{K} f_{i j}
$$

4.1.2. Center Frequency. The center frequency is the average instantaneous frequency weighted by the instantaneous

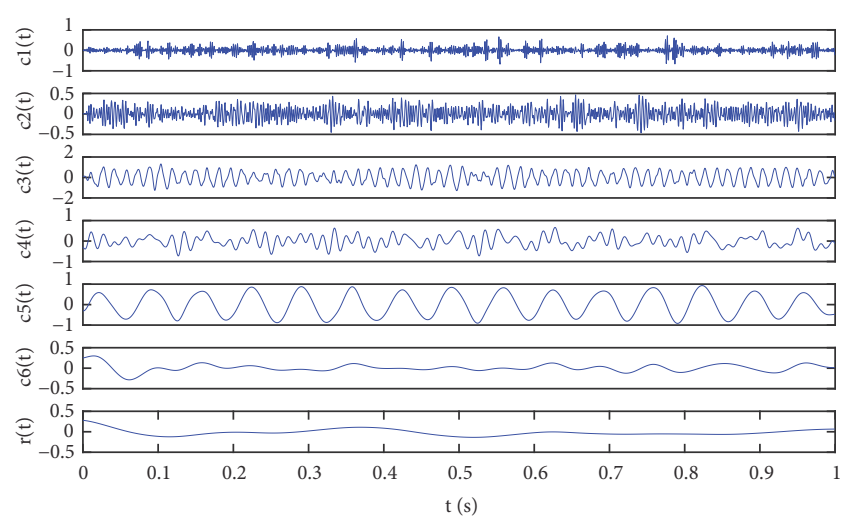

(b)

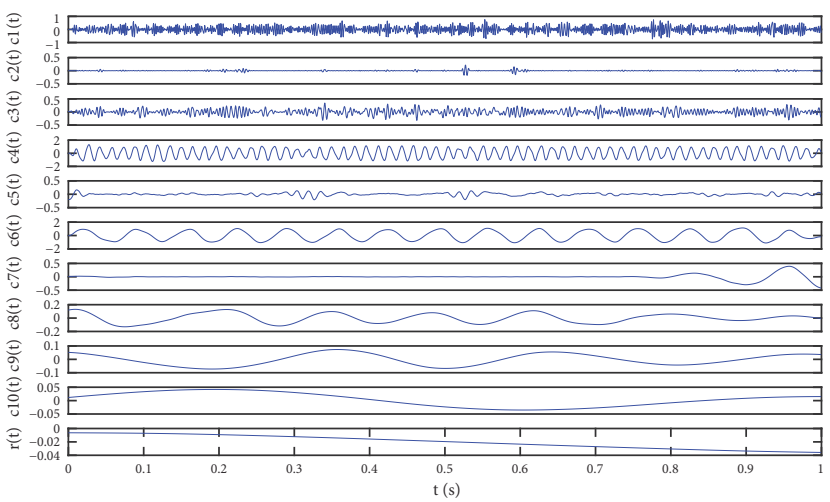

(d)

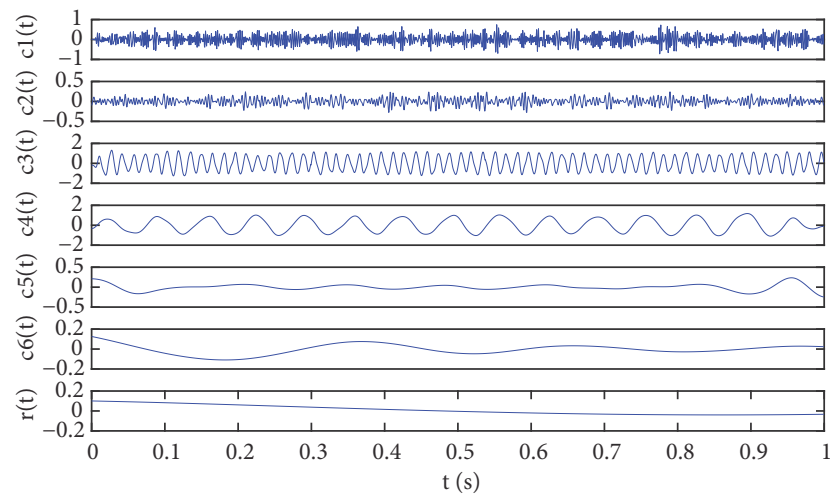

(f)

amplitude per unit time. The instantaneous amplitude $A_{i j}$ at the moment of $j$ is calculated after Hilbert transforming the $i$ th mode. Then the center frequency is defined as

$$
\overline{f_{m i}}=\frac{\sum_{j=1}^{K}\left|A_{i j}\right| f_{i j}}{\sum_{j=1}^{K}\left|A_{i j}\right|}
$$




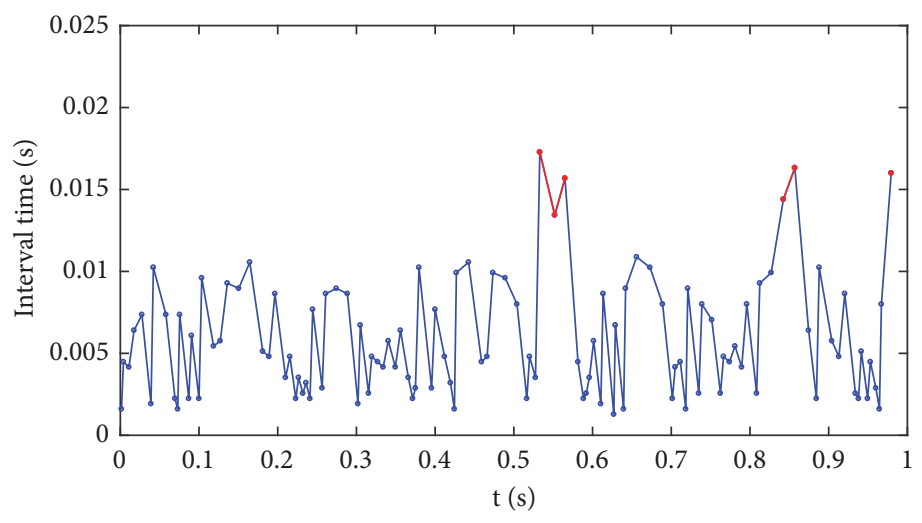

- High frequency intermittent component

- Low frequency intermittent component

(a)

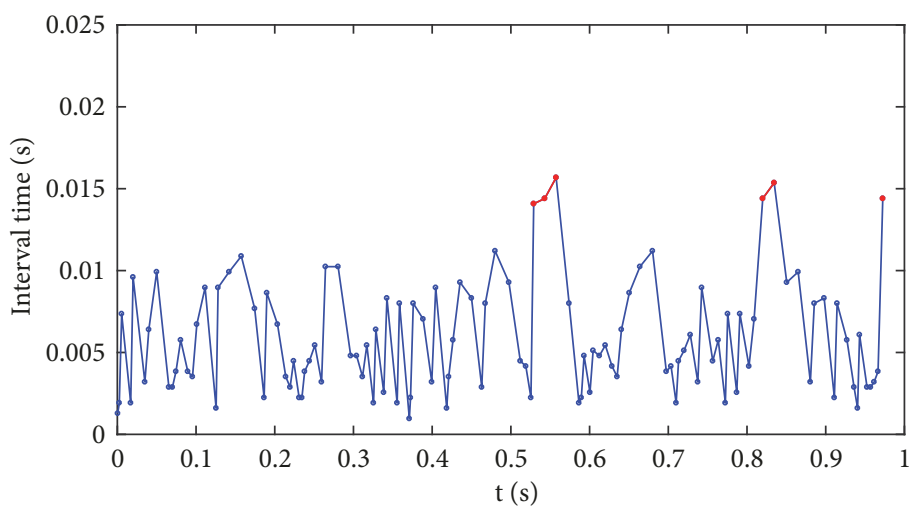

- High frequency intermittent component

- Low frequency intermittent component

(b)

Figure 8: Interval time between (a) maxima and (b) minima.

4.1.3. Energy Density. The energy density reflects the average energy of the mode per unit time. The energy density is defined as

$$
\overline{E_{i}}=\frac{1}{K} \sum_{j=1}^{K} A_{i j}^{2}
$$

4.1.4. Energy Distribution Ratio. The energy distribution ratio $E p_{i}$ describes the energy share of each mode. The ratio of the energy of the $i$ th mode to the energy of the original signal is defined as the energy distribution ratio with the expression:

$$
E p_{i}=\frac{\sum_{j=1}^{K} A_{i j}^{2}}{\sum_{i=1}^{N} \sum_{j=1}^{K} A_{i j}^{2}}
$$

4.2. Mode Feature Extraction. Two sets of ship radiated noise data are collected from a large ship named "Target A," and other two sets are collected from a boat and a yacht, which are named "Target B" and "Target C," respectively. Figure 11 shows the first 8 modes decomposed by SN-EMD from the four sets of data of the three targets after $5 \mathrm{kHz}$ low-pass filtering and normalization. The sampling rate is $48 \mathrm{kHz}$ and the data length is 9000 (0.1875 seconds). The average instantaneous frequency, center frequency, energy density, and energy distribution ratio are extracted from the modes, whose separability is verified in three-dimensional vector space.

The mean variances of normalized interval time between the maxima and minima of Target $A_{1}$ 's signal and first 7 residues are $0.019,0.022,0.066,0.019,0.018,0.018,0.024$, and 0.027 . So only the second-order residue contains intermittent component. The mean variances of other targets will not be listed here.

It can be seen that the amplitude of each mode is greatly reduced after the fourth-order mode in Figure 11, so the energy is mainly concentrated in the first four modes. Instantaneous phase curves over time of the first eight modes $(\mathrm{cl} \sim \mathrm{c} 8)$ of Target A' radiated noise are shown in Figure 12. Calculate the derivative of the instantaneous phase and divide it by $2 \pi$, so as to get instantaneous frequency. The instantaneous phase of the first four modes almost linearly 


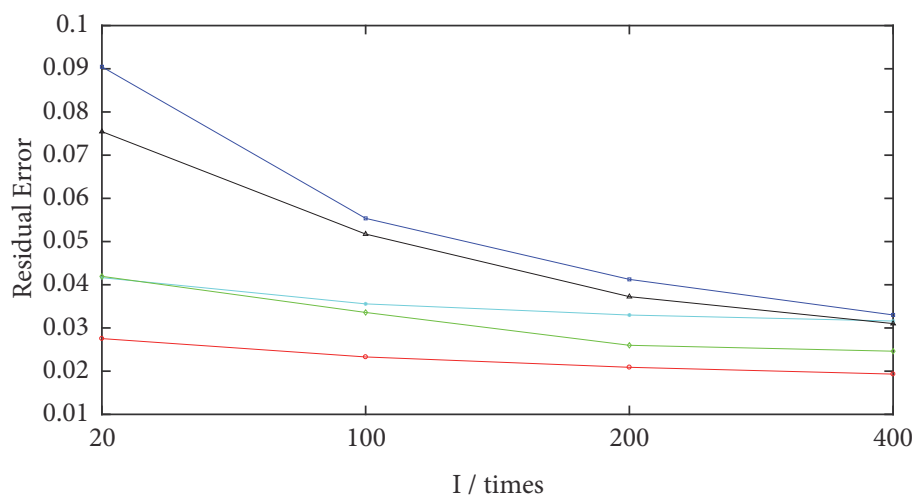

- EEMD

$\rightarrow$ CEEMD

- CEEMDAN

$\rightarrow$ Improved CEEMDAN

$\rightarrow$ SN-EMD

(a)

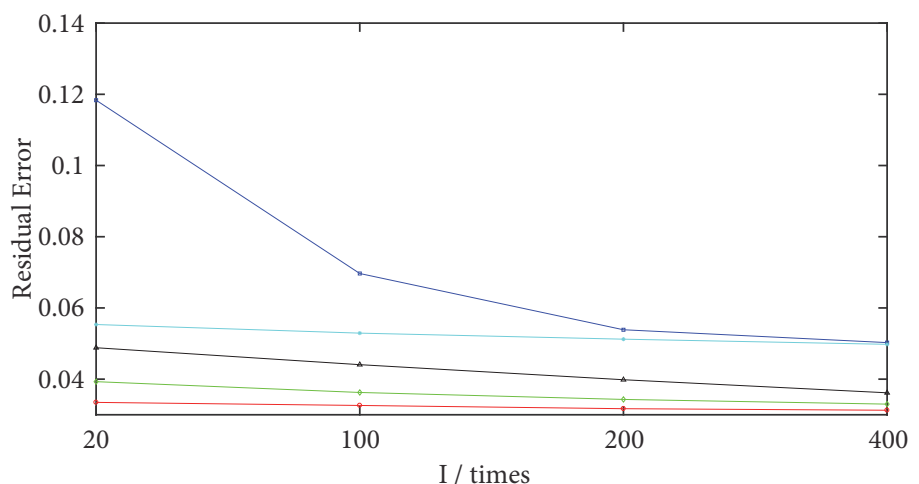

- EEMD

$\rightarrow$ CEEMD

- CEEMDAN

$\rightarrow$ Improved CEEMDAN

$\rightarrow$ SN-EMD

(b)

FIGURE 9: Residual error when recovering (a) $x_{1}(t)$ and (b) $x_{2}(t)$.

increases, indicating that the instantaneous frequency is relatively stable. Therefore, only the first four modes are processed below to extract feature quantities, which are shown by box-plot.

Instantaneous frequency and instantaneous amplitude are obtained for the first 4 modes. 30 average instantaneous frequencies and 30 central frequencies calculated by grouping and weighting average are shown in Figures 13 and 14. The horizontal axis coordinates in the figures correspond to the mode orders.

From Figures 13 and 14, it can be found that the average instantaneous frequency and center frequency of the same mode have no much difference. Target $\mathrm{A}_{1}$ has the mode frequency commensurate with Target $\mathrm{A}_{2}$. Comparing statistics of different targets, it can be seen that the first-order mode frequency of target $\mathrm{B}$ is higher than that of Target $\mathrm{A}$ and Target $\mathrm{C}$ about $400-500 \mathrm{~Hz}$, and the frequency of the second- to the fourth-order modes of target $\mathrm{C}$ is higher than that of Target A and Target B. That is, mode frequency of different targets has different distribution.

The energy density and energy distribution ratio of the first four modes of the four sets of data are counted as shown in Figures 15 and 16. The statistical results indicate that the first three modes' energies of Target A are not significantly different but are all higher than that of the fourth-order mode, while the mode energy of Target B is mainly concentrated in the first-order mode, with Target C's mode energy gradually decreasing. Among the three targets, the energy distribution of Target $\mathrm{A}$ is the most uniform, and the distribution of energy of Target $\mathrm{B}$ is the most uneven.

4.3. Separability Validation. Classifying and recognizing ship targets require that the mode feature of different targets should be distinguishable, while the mode feature of the 


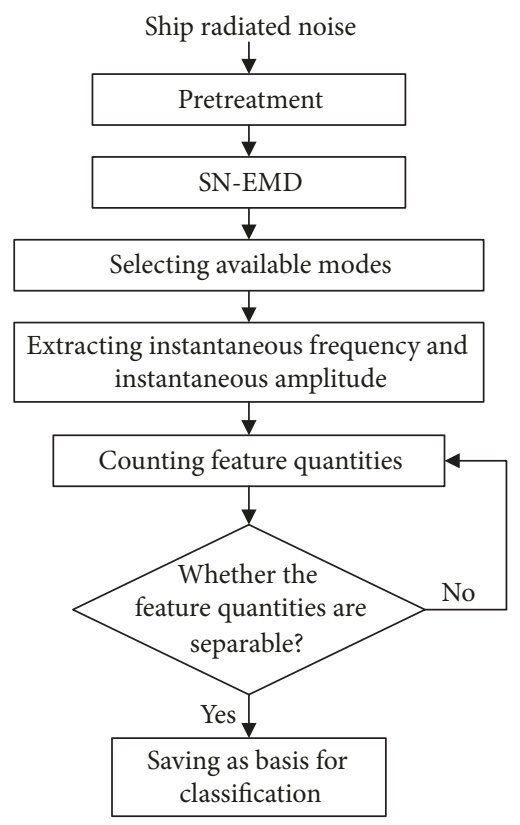

FIGURE 10: Flowchart of mode feature extraction.

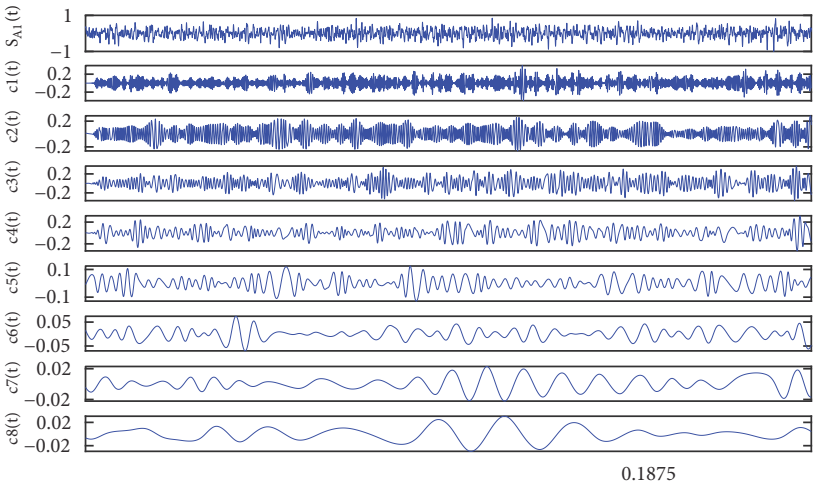

$t(s)$

(a)

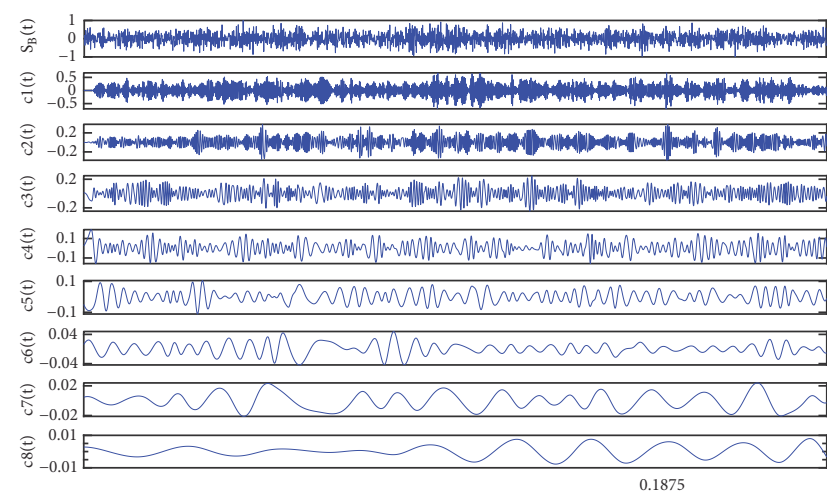

$t(s)$

(c)

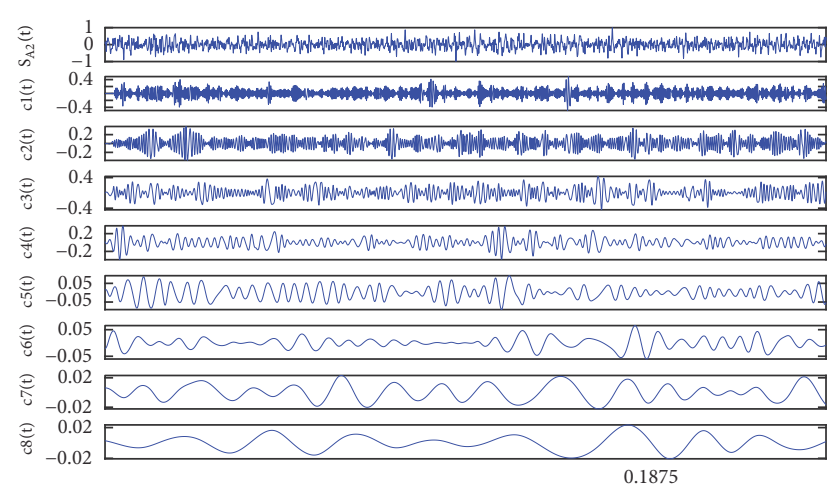

$t(s)$

(b)

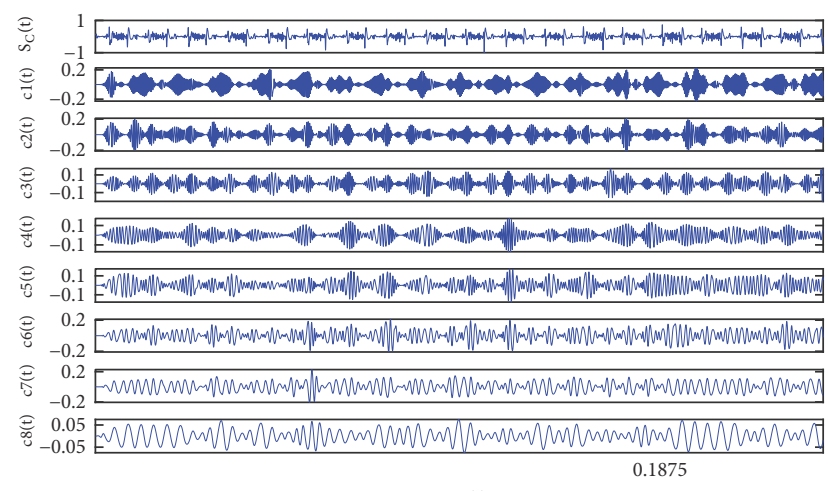

$\mathrm{t}(\mathrm{s})$

(d)

Figure 11: Decomposition of ship radiated noise signal (a) Target $A_{1}$; (b) Target $A_{2}$; (c) Target B; (d) Target C. 


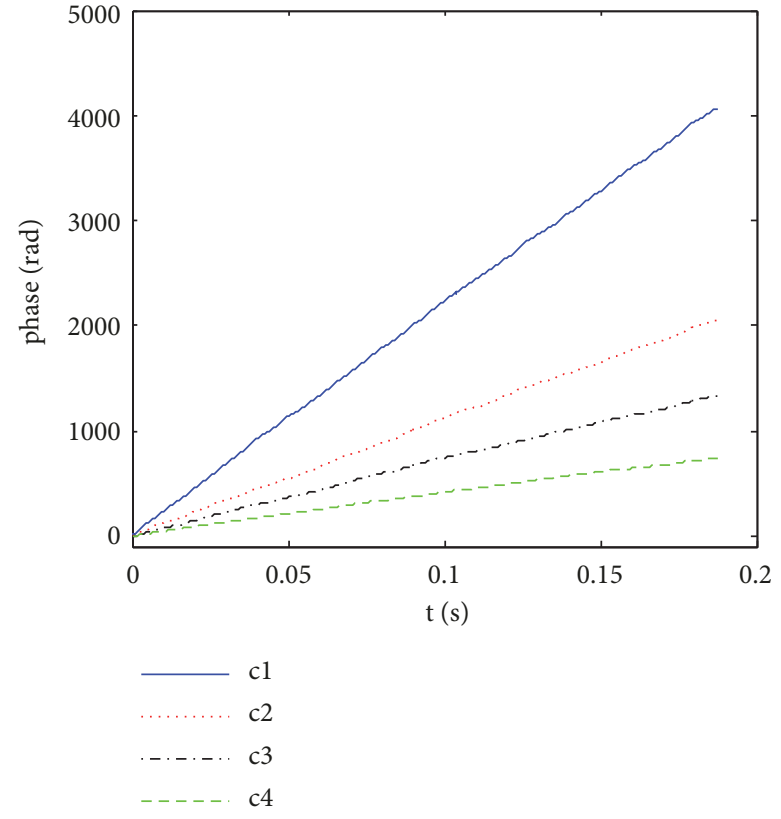

(a)

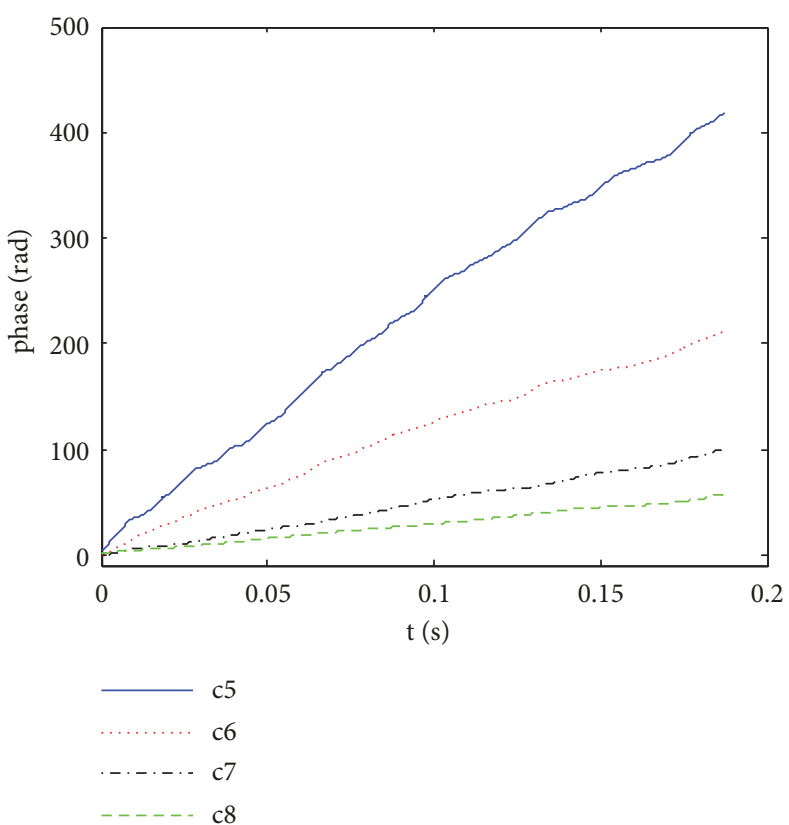

(b)

FIGURE 12: Instantaneous phase of first 8 modes of Target Al's radiated noise.
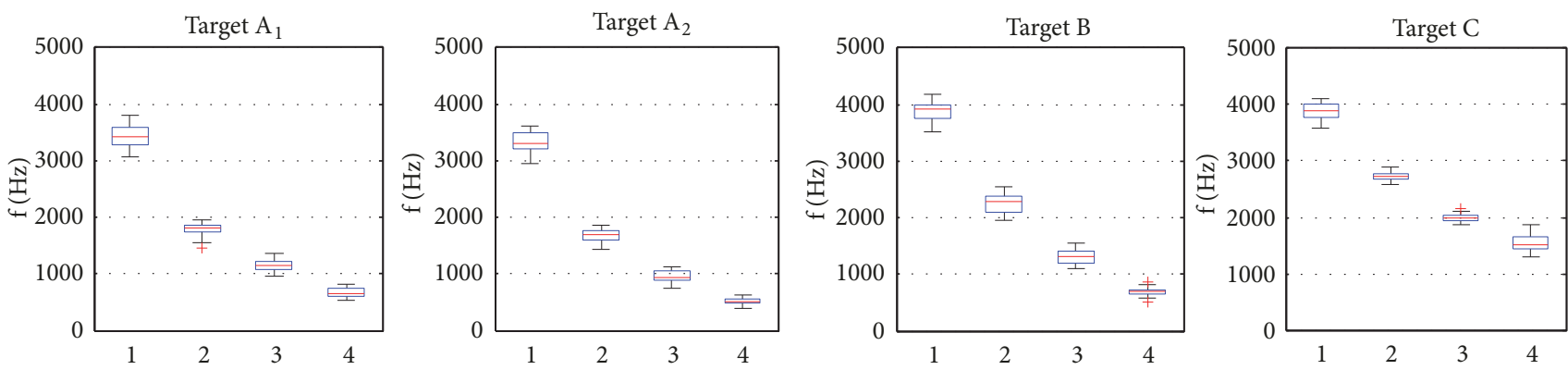

FIGURE 13: Statistics for average instantaneous frequency of first 4 modes.
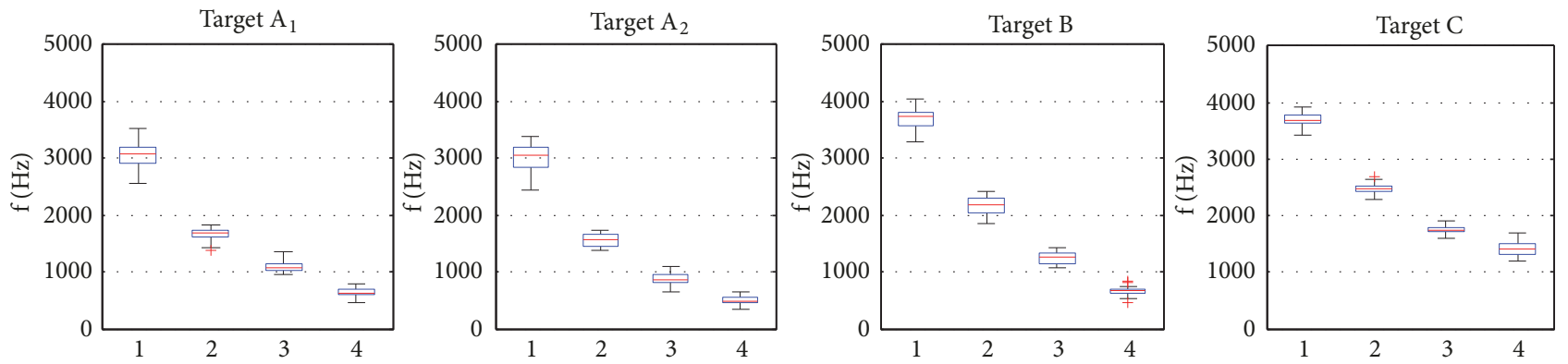

Figure 14: Statistics for center frequency of first 4 modes.

same target is similar. In order to verify the separability of average instantaneous frequency, center frequency, energy density, and energy distribution ratio feature, feature quantities of the first 3 modes extracted from the four sets of data are displayed in the three-dimensional space.
As shown in Figure 17, the three-dimensional coordinates, respectively, stand for the feature quantities of first three modes.

The feature quantities extracted from the two sets of data of Target A present mixed distribution in the same 

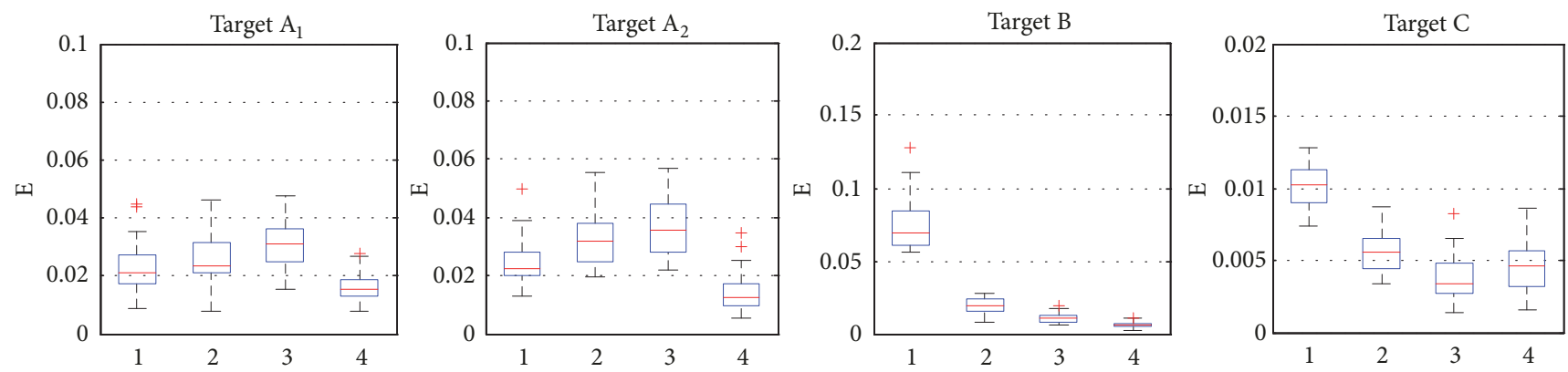

FIgURE 15: Statistics for energy density of first 4 modes.
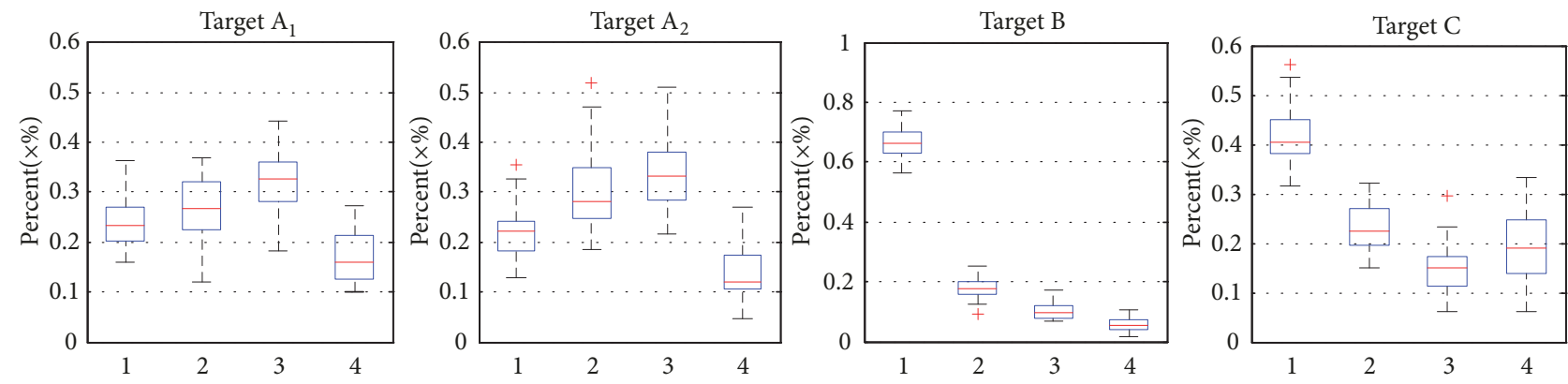

FIGURE 16: Statistics for energy distribution ratio of first 4 modes.

three-dimensional space range, while the feature quantities of the three targets mostly have different spatial distributions. This shows that the average instantaneous frequency, center frequency, energy density, and energy distribution ratio are easy to separate, and these feature quantities provide a basis for ship target classification and identification.

\section{Conclusions}

Based on the adaptive mode decomposition, the mode decomposition of ship radiated noise and mode feature extraction have been studied in this paper. Firstly, several existing mode decomposition algorithms were introduced, and the advantage and disadvantage were studied. Secondly, the SN-EMD algorithm was proposed to overcome the problems of the existing mode decomposition algorithms. Furthermore, the method of judging whether the signal contains intermittent component was given. By contrasting the performance parameters of these algorithms, the results showed that the new algorithm has better performance on resisting mode mixing and time-consuming than other noise-assisted algorithms, and residual error when recovering signal components and reconstruction error are also relatively minimal. Finally, based on the differences in the original information contained in each mode of different ships' radiated noise, the first 3 modes were selected to extract the average instantaneous frequency, center frequency, energy density, and energy distribution ratio. In the three-dimensional space, distribution of feature quantities verifies that features of the same target are similar and the features of different targets are separable. As a result, the feature quantities can be seen as the basis for ship classification and identification.

\section{Data Availability}

The data used to support the findings of this study are available from the corresponding author upon request.

\section{Conflicts of Interest}

The authors declare that there are no conflicts of interest regarding the publication of this paper.

\section{Acknowledgments}

This work was partially supported by the National Natural Science Foundation of China (Grant No. 61371171, 11374072, 61501061), Open Foundation of National Key Laboratory of Science and Technology on Underwater Acoustic 

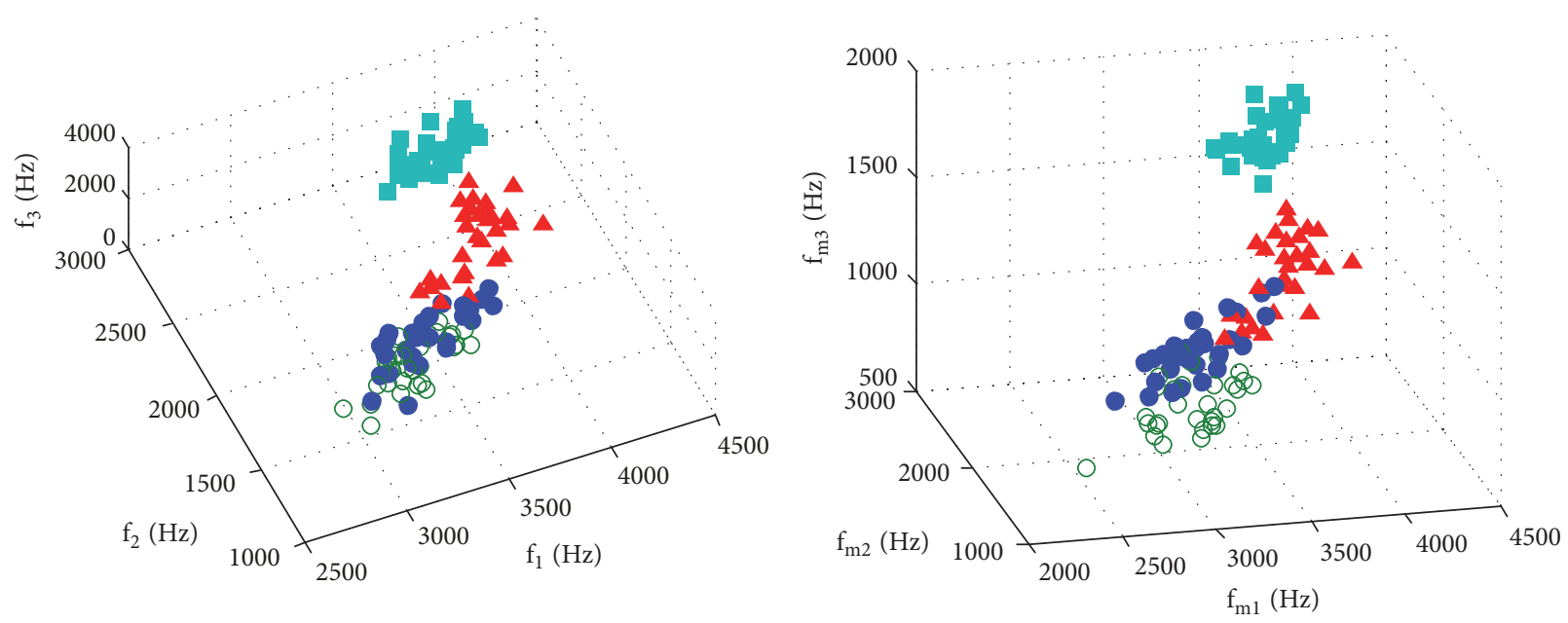

Target $\mathrm{A}_{1}$

$\bigcirc$ Target $\mathrm{A}_{2}$

- Target $\mathrm{B}$

Target $\mathrm{C}$

(a)

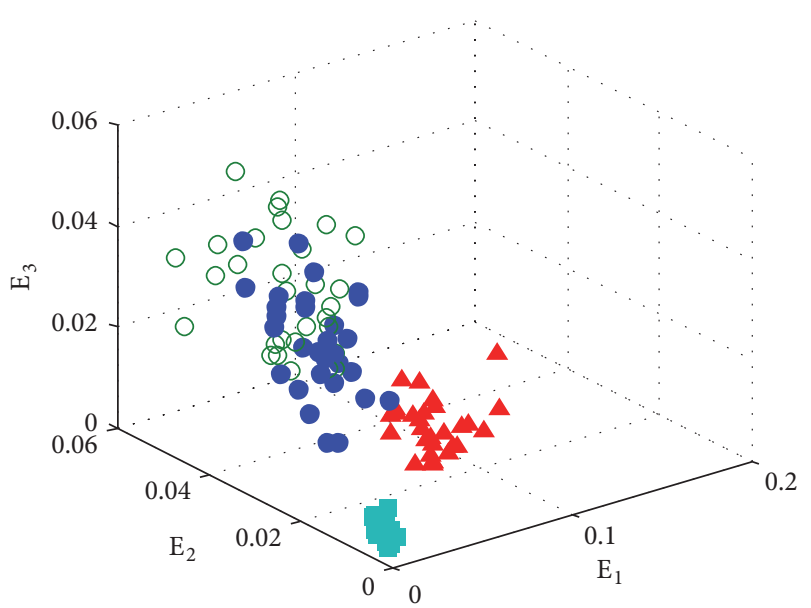

Target $A_{1}$

$\bigcirc$ Target $\mathrm{A}_{2}$

- Target $\mathrm{B}$

- Target $\mathrm{C}$

(b)

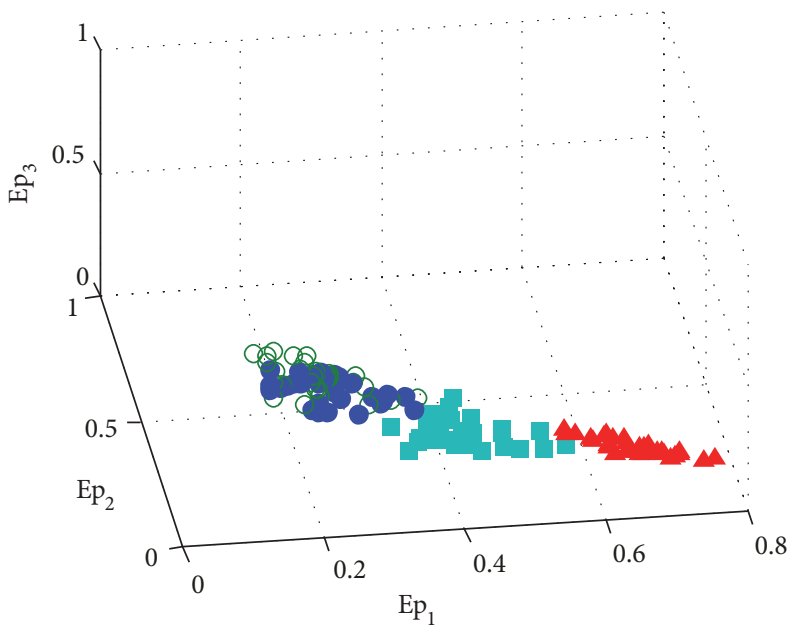

- Target $\mathrm{A}_{1}$

$\bigcirc$ Target $A_{2}$

- Target $\mathrm{B}$

- Target $\mathrm{C}$

(c)

(d)

FIGURE 17: Feature quantities of first 3 modes distributed in three-dimensional space: (a) average instantaneous frequency; (b) center frequency; (c) energy density; (d) energy distribution ratio.

Antagonizing (SSDKKFJJ-2017-02-01), and Acoustic Science and Technology Laboratory Stable Support Project (SSJSWDZC2018002).

\section{References}

[1] N. E. Huang, Z. Shen, S. R. Long, and M. C. Wu, "The empirical mode decomposition and the Hilbert spectrum for nonlinear and non-stationary time series analysis," Proceedings of the Royal Society of London A: Mathematical, Physical and Engineering Science, vol. 454, no. 1971, pp. 903-995, 1998.

[2] Z. H. Wu and N. E. Huang, "Ensemble empirical mode decomposition: a noise-assisted data analysis method," Advances in Adaptive Data Analysis (AADA), vol. 1, no. 1, pp. 1-41, 2009.

[3] J. Yeh, J. Shieh, and N. E. Huang, "Complementary ensemble empirical mode decomposition: a novel noise enhanced data analysis method," Advances in Adaptive Data Analysis (AADA), vol. 2, no. 2, pp. 135-156, 2010. 
[4] M. E. Torres, M. A. Colominas, G. Schlotthauer, and P. Flandrin, "A complete ensemble empirical mode decomposition with adaptive noise," in Proceedings of the 36th IEEE International Conference on Acoustics, Speech, and Signal Processing, pp. 41444147, May 2011.

[5] M. A. Colominas, G. Schlotthauer, and M. E. Torres, "Improved complete ensemble EMD: a suitable tool for biomedical signal processing," Biomedical Signal Processing and Control, vol. 14, no. 1, pp. 19-29, 2014.

[6] Z. Xu and T. Liu, "Vital sign sensing method based on EMD in terahertz band," EURASIP Journal on Advances in Signal Processing, vol. 2014, no. 1, p. 75, 2014.

[7] A. Hooshmand, J. Nasseri, and H. R. Siahkoohi, "Seismic data de-noising based on the complete ensemble empirical mode decomposition," in Proceedings of the International Geophysical Conference and Oil and Gas Exhibition, pp. 17-19, 2012.

[8] J. Han and V. D. B. Mirko, "Empirical mode decomposition for seismic time-frequency analysis," Geophysics, vol. 78, no. 2, pp. 9-19, 2013.

[9] L. Bai, J. Xu, Z. Chen et al., "The regional features of temperature variation trends over Xinjiang in China by the ensemble empirical mode decomposition method," International Journal of Climatology, vol. 35, no. 11, pp. 3229-3237, 2015.

[10] L. G. N. Martins, S. D. Miller, and O. C. Acevedo, "Using Empirical Mode Decomposition to Filter Out Non-turbulent Contributions to Air-Sea Fluxes," Boundary Layer Meteorology, vol. 163, no. 1, pp. 123-141, 2017.

[11] D. Camarena-Martinez, M. Valtierra-Rodriguez, C. A. PerezRamirez, J. P. Amezquita-Sanchez, R. De Jesus RomeroTroncoso, and A. Garcia-Perez, "Novel downsampling empirical mode decomposition approach for power quality analysis," IEEE Transactions on Industrial Electronics, vol. 63, no. 4, pp. 2369-2378, 2016.

[12] S. Shukla, S. Mishra, and B. Singh, "Power quality event classification under noisy conditions using EMD-based de-noising techniques," IEEE Transactions on Industrial Informatics, vol. 10, no. 2, pp. 1044-1054, 2014.

[13] Y. Li, P. W. Tse, X. Yang, and J. Yang, "EMD-based fault diagnosis for abnormal clearance between contacting components in a diesel engine," Mechanical Systems and Signal Processing, vol. 24, no. 1, pp. 193-210, 2010.

[14] D. Yu, J. Cheng, and Y. Yang, "Application of EMD method and Hilbert spectrum to the fault diagnosis of roller bearings," Mechanical Systems and Signal Processing, vol. 19, no. 2, pp. 259270, 2005.

[15] C. Damour, M. Benne, B. Grondin-Perez, M. Bessafi, D. Hissel, and J.-P. Chabriat, "Polymer electrolyte membrane fuel cell fault diagnosis based on empirical mode decomposition," Journal of Power Sources, vol. 299, pp. 596-603, 2015.

[16] F. Bao, C. Li, X. Wang, Q. Wang, and S. Du, "Ship classification using nonlinear features of radiated sound: An approach based on empirical mode decomposition," The Journal of the Acoustical Society of America, vol. 128, no. 1, pp. 206-214, 2010.

[17] A. T. Çelebi and S. Ertürk, "Visual enhancement of underwater images using Empirical Mode Decomposition," Expert Systems with Applications, vol. 39, no. 1, pp. 800-805, 2012.

[18] R. J. Urick, Principles of Underwater Sound, Harbin Shipbuilding Engineering Institute Press, Harbin, China, 1990.

[19] P. Flandrin, G. Rilling, and P. Gonçalvés, "Empirical mode decomposition as a filter bank," IEEE Signal Processing Letters, vol. 11, no. 2, pp. 112-114, 2004.
[20] N. Rehman and D. P. Mandic, "Filter bank property of multivariate empirical mode decomposition," IEEE Transactions on Signal Processing, vol. 59, no. 5, pp. 2421-2426, 2011. 


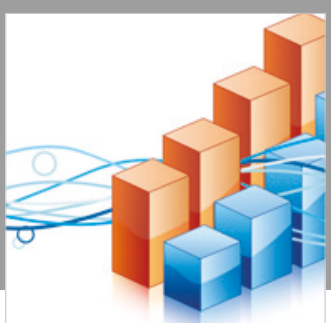

Advances in

Operations Research

\section{-n-m}
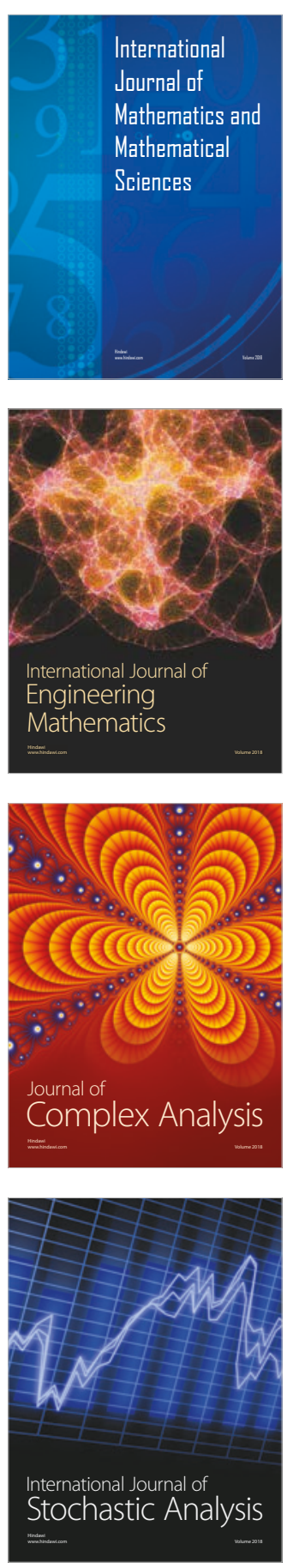
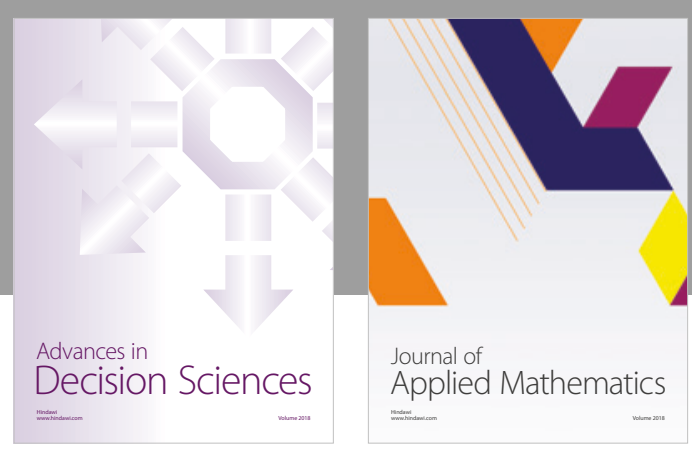

Journal of

Applied Mathematics
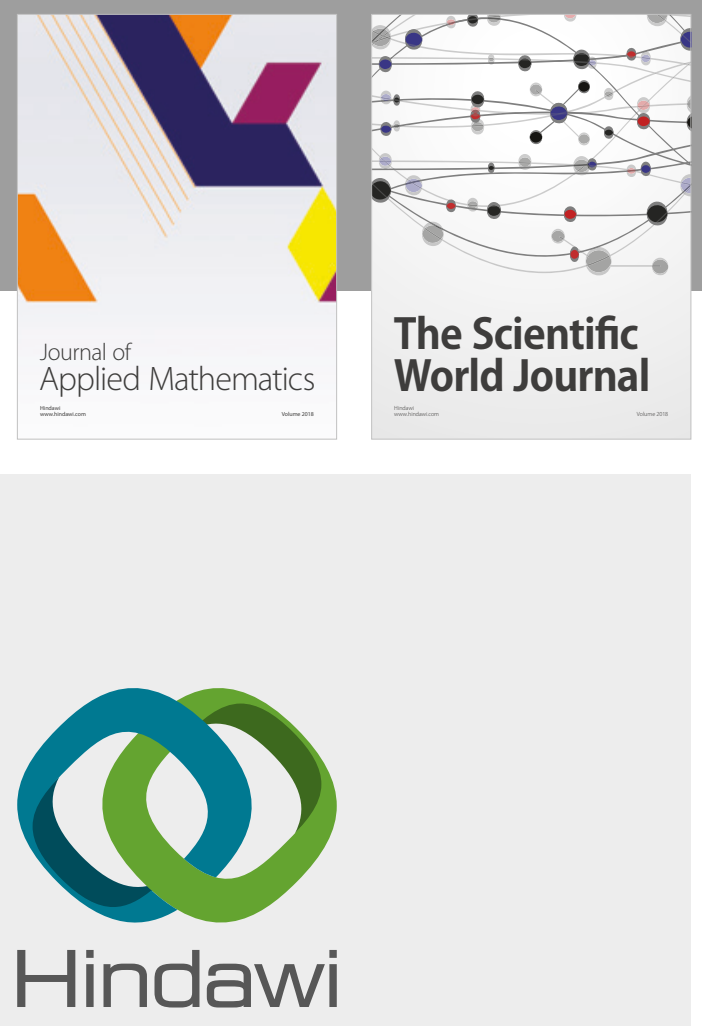

Submit your manuscripts at

www.hindawi.com

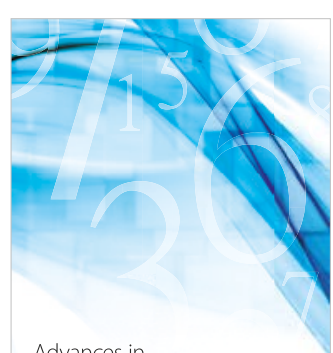

Advances in
Numerical Analysis
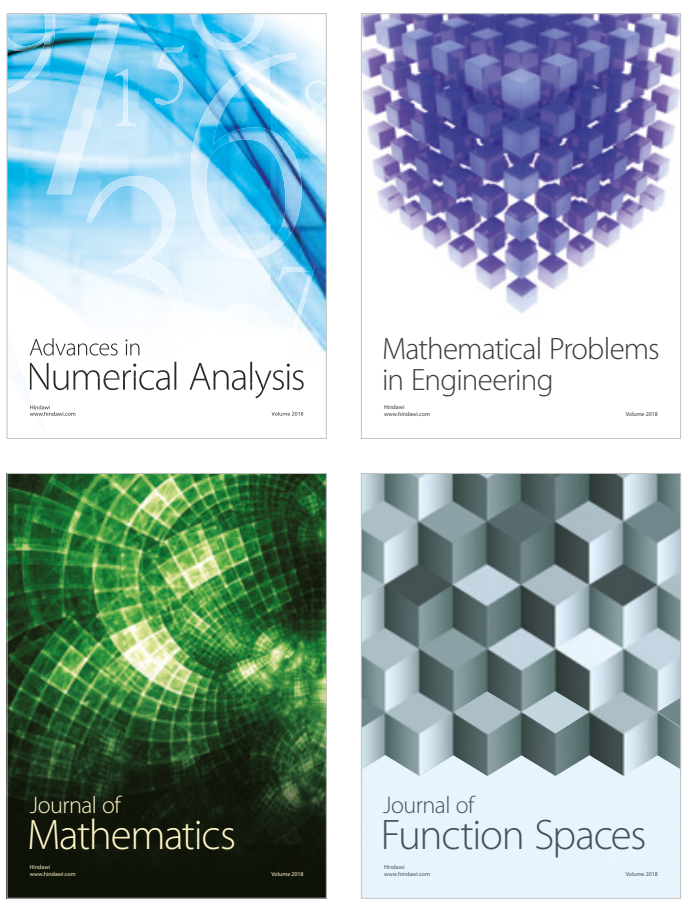

Mathematical Problems in Engineering

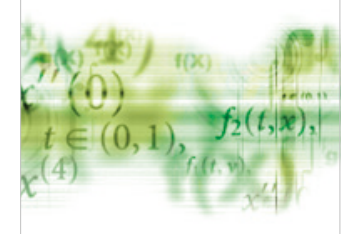

International Journal of

Differential Equations

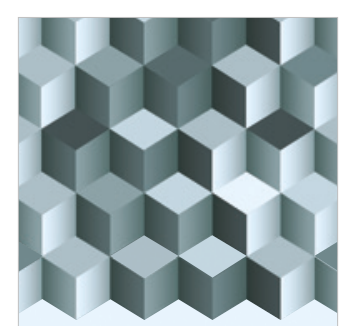

Journal of

Function Spaces

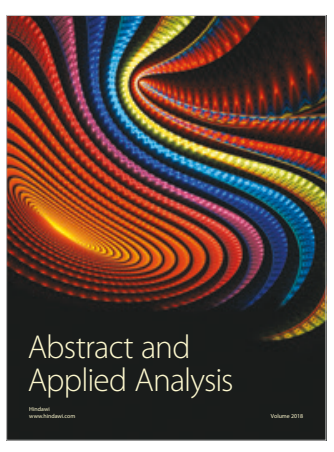

The Scientific

World Journal

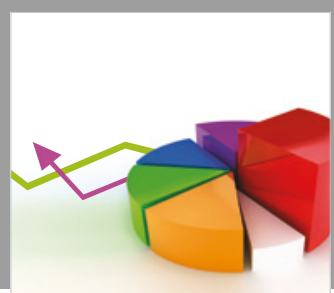

Journal of

Probability and Statistics
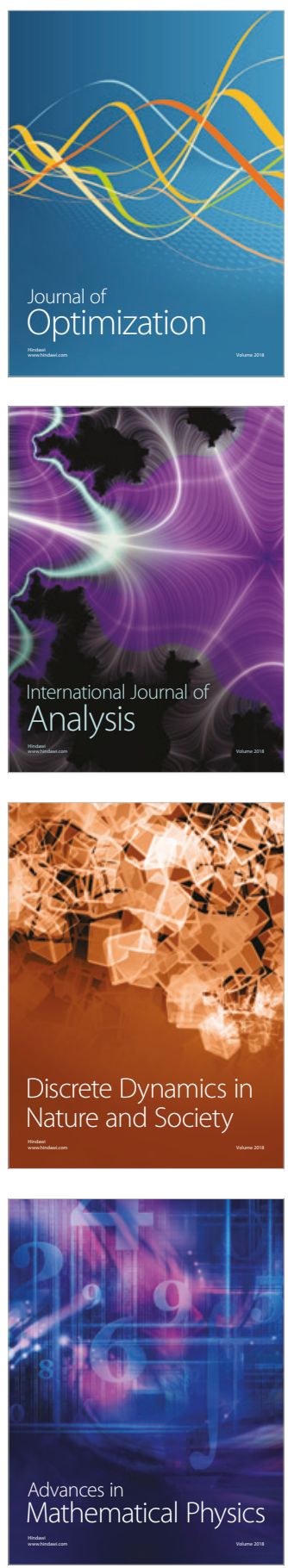